\title{
Der Vertrag von Lissabon auf der Systemspur des Europäischen Primärrechts
}

\author{
Peter-Christian Müller-Graff*
}

Der Vertrag von Lissabon ${ }^{1}$ auf der Systemspur des Europäischen Primärrechts ${ }^{2}$ - bereits eine derartige thematische Eintaktung ist vielleicht überraschend. Gestaltet denn der am 13. Dezember 2007 in Lissabon von den Staats- und Regierungschefs der 27 Mitgliedstaaten der Europäischen Gemeinschaft und Union unterzeichnete Reformvertrag, so mag man fragen, nicht selbst das System des Europäischen Primärrechts, sobald er von allen Mitgliedstaaten ratifiziert ist? Ist er also nicht selbst Systemgeber, keineswegs aber Systemunterworfener? Oder noch zugespitzter gefragt: Gibt es eine stärkere rechtspolitische Systemautorität als die ,Herren der Verträge', mithin die Mitgliedstaaten?

Die Grundthese dieses Beitrags zu dieser Frage mag daher vielleicht erstaunen: $\mathrm{Ob}-$ wohl die Mitgliedstaaten vor (und auch nach dem Reformvertrag ${ }^{3}$ ) zweifellos die Gestaltungshoheit haben, sind sie doch nachhaltig systemgebunden. Denn sie haben diese Gestaltungshoheit nur in Einstimmigkeit. ${ }^{4}$ Dadurch gewinnt aber die Pfadabhängigkeit von den Grundkonturen des Acquis des Primärrechts überragendes Gewicht: mithin vom Bestand, wie er sich seit den Gründungsverträgen der Europäischen Gemeinschaften allmählich entwickelnd über nunmehr fünfzehn Hauptreformverträge aufgeschichtet hat, ${ }^{5}$ darunter als wichtigste die Einheitliche Europäische Akte (1986), der Vertrag von Maastricht (1992), der Vertrag von Amsterdam (1997) und der Vertrag von Nizza (2001). Die Pfadabhängigkeit vom Acquis zeigte sich schon im Inhalt des Verfassungsvertrages

Prof. Dr. Dr. h.c. Peter-Christian Müller-Graff, Universität Heidelberg.

1 Vertrag von Lissabon zur Änderung des Vertrags über die Europäische Union und des Vertrags zur Gründung der Europäischen Gemeinschaft vom 13. Dezember 2007, in: Amtsblatt der Europäischen Union, Nr. C 306 vom 17. Dezember 2007, S. 1. In der zukünftigen konsolidierten Version werden die Artikel der beiden Verträge nach Art. 5 Abs. 1 des Vertrags von Lissabon gemäß den Übereinstimmungstabellen im Anhang des Vertrags neu nummeriert. Auf diese projektierte Neunummerierung der Artikel wird im Folgenden jeweils hingewiesen. Als erste inoffizielle konsolidierte Fassung beider Verträge einschließlich der Protokolle und Erklärungen vgl. die Textfassung durch Klemens H. Fischer: Der Vertrag von Lissabon, Baden-Baden 2008, S. 103-523. Als eine erste politikwissenschaftliche Würdigung vgl. Andreas Hofmann/Wolfgang Wessels: Der Vertrag von Lissabon - eine tragfähige und abschließende Antwort auf konstitutionelle Grundfragen?, in: integration 1/2008, S. 3-20.

2 Scil.: Acquis des Vertragsrechts des Vertrags zur Gründung der Europäischen Gemeinschaft (EGV) und des Vertrags über die Europäische Union (EUV). Der Text dieses Beitrags beruht im zweiten Teil auf einem Vortrag, den der Verfasser am 18. Januar 2008 vor dem Europäischen Graduiertenkolleg der Deutschen Forschungsgemeinschaft „Systemtransformation und Wirtschaftsintegration im zusammenwachsenden Europa“ der Universitäten Heidelberg, Krakau, Mainz an der Jagiellonen-Universität in Krakau gehalten hat.

3 Auch die beiden vom Reformvertrag eingeführten vereinfachten Änderungsverfahren (projektierter neuer Art. 48 Abs. 6 und 7 EUV) beschränken die Mitgliedstaaten nicht in ihrem Recht, die von Art. 48 Abs. 6 und 7 EUV geregelten Bereiche im ordentlichen Änderungsverfahren des projektierten neuen Art. 48 Abs. 2-5 EUV zu behandeln, mithin entweder zu ändern oder Änderungen, die in einem vereinfachten Änderungsverfahren vorgenommen wurden, zu ändern.

4 Vgl. derzeit Art. 48 Abs. 3 EUV; in der Perspektive des Reformvertrags der projektierte neue Art. 48 Abs. 4 S. 2, Abs. 6 UAbs. 2 S. 3, Abs. 7 UAbs. 3 (mit der Besonderheit der Nicht-Ablehnung durch ein nationales Parlament) EUV.

5 Vgl. Peter-Christian Müller-Graff: Primärrechtliche Entwicklungsschritte der Gemeinschaftsintegration zu einem transnationalen Gemeinwesen, in: integration 4/2007, S. 407-421. 
(VVE). ${ }^{6}$ Denn entgegen verbreiteter, durch verkürzte Redeweise geförderter Fehlverständnisse beinhaltete er keinen Umsturz in der normativen Grundschrift des gewachsenen transnationalen europäischen Gemeinwesens. Vielmehr verschmolz er das in einem halben Jahrhundert gewachsene Primärrecht von Europäischer Gemeinschaft und Europäischer Union zu einem großen Kodifikationsangebot, systematisierte es und entwickelte es im Interesse von Legitimations- und Effizienzsteigerung systemrational fort. ${ }^{7}$ Auch das Mandat des Europäischen Rates vom 21. Juni 2007 zur Rettung der wesentlichen Neuheitensubstanz des Verfassungsvertrages folgte dieser Linie. ${ }^{8}$ Der nun auf dieser Grundlage erarbeitete Vertrag von Lissabon (VvL) beinhaltet gleichermaßen keine Systemrevolution. Vielmehr modernisiert er das bestehende System des Primärrechts für die erweiterte Union und deren globale Rolle auf der Spur der nunmehr anders verpackten Neuerungen des Verfassungsvertrages, ohne es aber zu sprengen. Diese Grundeinschätzung ist unter der Leitfrage nach Neuerungen und Kontinuität in zwei Teilen aufzufächern: Zunächst sind die wesentlichen Grundlinien der Neuerungen zu skizzieren und sodann ist der VvL auf sein Verhältnis zu den wesentlichen primärrechtlichen Systemkonturen der Gemeinschaftsintegration in ein transnationales Gemeinwesen sowie auf neue Systemkonturen auf diesem Weg zu befragen.

\section{Die wesentlichen Grundlinien der Neuerungen im Verhältnis zum Acquis und zum Verfassungsvertrag}

In seinen wesentlichen Grundlinien der Neuerungen weicht der Lissabonner Vertrag nur unwesentlich von denen des Verfassungsvertrags ab und entwickelt dadurch das geltende Primärrecht signifikant fort.

\section{Hauptziele des Reformvertrags}

In seinen Hauptzielen richtet sich der Reformvertrag anders als der Verfassungsvertrag nicht auf die Gründung einer rechtlich neuen supranationalen Organisation auf der Grundlage eines neuen Vertrages unter Aufhebung der bestehenden Verträge (so Art. IV-437 VVE). Vielmehr verwirklicht der VvL das schon vom VVE verfolgte Ziel des Zusammenziehens von Gemeinschaft und Union nunmehr mittels der an das Gesellschaftsrecht ${ }^{9}$ erin-

$6 \mathrm{Zu}$ dessen Grundlinien Peter-Christian Müller-Graff: Strukturmerkmale des neuen Verfassungsvertrages für Europa im Entwicklungsgang des Primärrechts, in: integration 3/2004, S. 186-201; Heribert Franz Jöck/Tanja Marktler: Der Verfassungsvertrag - Überblick und Analyse, in: Klaus Beckmann/Jürgen Dieringer/Ulrich Hufeld (Hrsg.): Eine Verfassung für Europa, 2. Aufl., Tübingen 2005, S. 329-358; aus politikwissenschaftlicher Sicht vgl. Wolfgang Wessels: Die institutionelle Architektur der EU nach der Europäischen Verfassung: Höhere Entscheidungsdynamik - neue Koalitionen?, in: integration 3/2004, S. 161-175; als Würdigung des Verfassungsentwurfs des Europäischen Konvents vgl. Wolfgang Wessels: Der Verfassungsvertrag im Integrationstrend: Eine Zusammenschau zentraler Ergebnisse, in: integration 4/2003, S. 284-300; als sichtwinkelreiche und umfängliche pluridisziplinäre Einbettung des Verfassungsvertrags in die Diskussion um die Konstitutionalisierung Europas vgl. Beckmann/Dieringer/Hufeld (Hrsg.): Eine Verfassung für Europa, 2005.

7 Vgl. zur Systemrationalität Peter-Christian Müller-Graff: Systemrationalität in Kontinuität und Änderung des Europäischen Verfassungsvertrags, in: integration 4/2003, S. 301-316; Peter-Christian Müller-Graff: Eine neue Verfassung für Europa. Kriterien der Europarechtswissenschaft, in: Beckmann/Dieringer/Hufeld (Hrsg.): Eine Verfassung für Europa, 2005, S. 311-328.

8 Vgl. dazu Peter-Christian Müller-Graff: Die Zukunft des europäischen Verfassungstopos und Primärrechts nach der deutschen Ratspräsidentschaft, in: integration 3/2007, S. 223-237; Andrew Duff: Das Mandat für die Regierungskonferenz - Die zweite Chance, in: integration 3/2007, S. 333-337; zu der vorlaufenden ,Berliner Erklärung‘ vom 25.03.2007 vgl. Thomas Oppermann: Die Berliner Erklärung vom 25. März 2007, in: Jörn Ipsen/Bernhard Stüer (Hrsg.): Europa im Wandel. Festschrift für Hans-Werner Rengeling, Köln/München 2008, S. 609ff.; Timo Goosmann: Die ,Berliner Erklärung ‘ - Dokument europäischer Identität oder pragmatischer Zwischenschritt zum Reformvertrag?, in: integration 3/2007, S. 251-263.

9 Vgl. zu den Formen der Verschmelzung im privaten Verbandsrecht die Modelle in den Regelungen der $\S 2 \mathrm{ff}$. des Umwandlungsgesetzes vom 28. Oktober 1994 (BGB1. I S. 3210, ber. BGB1. I 1995, S. 428). 
nernden, wiewohl nicht exakt parallelisierenden Methode der Verschmelzung der rechtspersönlichkeitslosen Union auf die rechtspersonale Gemeinschaft zu einem einzigen Verband mit ungeteilter Rechtspersönlichkeit unter dem einheitlichen Namen „Europäische Union“ (Art. 1 Ziff. $55 \mathrm{VvL}^{10}$ ). Dies geschieht allerdings auf der Grundlage von zwei Verträgen: dem Vertrag über die Europäische Union (EUV; nunmehr maßvoll erweitert auf 55 Artikel) und dem aus dem derzeitigen EGV hervorgehenden Vertrag über die Arbeitsweise der Europäischen Union (AV; nunmehr erweitert auf 358 Artikel) sowie der vertragsgleich gestellten Grundrechte-Charta und den beigefügten Protokollen.

Wie der Verfassungsvertrag zielt der VvL auf die zukunftsfähige Anpassung des rechtsverbindlichen Gerüsts der nunmehr auf 27 Mitgliedstaaten erweiterten Union an die Herausforderungen und Chancen dieser Erweiterung ebenso wie an diejenigen einer sich dichter vernetzenden Welt. Der Reformvertrag projektiert hierbei, grobstrichig gesprochen, auf der Grundlage grundsätzlicher rechtskonzeptioneller Kontinuität eine Steigerung des Potenzials der inneren und äußeren Handlungsfähigkeit und eine Erhöhung der Legitimation unionaler Maßnahmen, wohingegen die vom Verfassungsvertrag projektierte Texttransparenz und Staatsanalogisierung teils zurückgeschnitten werden und die bisherige konzeptionelle Klarheit im operativen primärrechtlichen Acquis Einbußen erleidet und einer konzeptionellen Komplexierung Raum gibt.

\section{Rechtskonzeptionelle Kontinuität}

In seiner rechtskonzeptionellen Grundanlage beinhaltet der VvL, wie schon der VVE, keinen normativen Umsturz. Er bildet vielmehr das seit einem halben Jahrhundert erwachsene Grundkonzept systemrational weiter ${ }^{11}$ und lässt die Finalitätsfrage der unionalen Form klugerweise offen. Der Reformvertrag belässt nicht anders als der VVE den europäischen Verband auf dem historisch und kategorial beispiellosen Pfad der Gemeinschaftsintegration in ein transnationales Gemeinwesen ${ }^{12}$ und befestigt diesen weiter.

Völkervertragliche Grundlegung: Dies zeigt sich erstens darin, dass es bei dessen völkervertraglicher Grundlegung (Ratifikationserfordernis gemäß Art. 6 VvL) und deren Konsequenzen bleibt. Wie schon vom VVE wird der Rubikon zu einem sich selbst konstituierenden Gemeinwesen nicht überquert. Nichts anderes gilt für die Ausgestaltung künftiger Vertragsänderungen. ${ }^{13}$ Sie übernehmen die schon im VVE vorgesehenen Neuerungen ${ }^{14}$ mit der Zweiteilung in ein ordentliches (regelmäßig über Konvent, Regierungskonferenz und mitgliedstaatliche Ratifikationen laufendes) Änderungsverfahren ${ }^{15}$ und zwei vereinfachte (über Europäischen Rat und entweder mitgliedstaatliche Zustimmungen oder nationale Parlamente laufende) Änderungsverfahren. ${ }^{16}$ Auch letztere können aber zu einer primärrechtlichen Änderung gegen den Willen eines Mitgliedstaats nicht führen, wobei es freilich zu einer bemerkenswerten Differenzierung kommt, auf wessen politische Willensartikulation in einem Mitgliedstaat es ankommt (bei der vereinfachten Änderung von Bestimmungen im Teil der internen Politiken und Maßnahmen der Union: Zustimmung im Einklang mit den

10 Art. 46a EUV (projektierte Neunummerierung: Art. 47 EUV).

11 Dies liegt auf der Linie des Verfassungsvertrages; vgl. dazu Müller-Graff: Systemrationalität, 2003; MüllerGraff: Eine neue Verfassung für Europa, 2005.

12 Vgl. zu den primärrechtlich herausragend systembildenden Einzelmerkmalen auf diesem Weg Müller-Graff: Primärrechtliche Entwicklungsschritte der Gemeinschaftsintegration, 2007.

13 Vgl. Art. 1 Ziff. 56 VvL: Art. 48 EUV = projektierter neuer Art. 48 EUV.

14 Dazu Müller-Graff: Strukturmerkmale des neuen Verfassungsvertrages, 2004, S. 189-190.

15 Vgl. den projektierten neuen Art. 48 Abs. 2-5 EUV; vgl. dazu Art. IV-443 VVE.

16 Vgl. den projektierten neuen Art. 48 Abs. 6-7 EUV; vgl. dazu Artt. IV-445 und IV-444 VVE. 
verfassungsrechtlichen Vorschriften $;{ }^{17}$ bei definierten Übergängen im Entscheidungsverfahren: keine Ablehnung durch ein nationales Parlament ${ }^{18}$ ). Neuartig pointiert wird die staatengetragene Grundlage durch Rezeption des schon vom VVE vorgesehenen ausdrücklichen Austrittsrechts, das an keine Voraussetzung gebunden ist. ${ }^{19}$

Gesellschaftsvertragsartige Zielbindung: Zweitens zeigt sich die konzeptionelle Kontinuität auch in der Fortsetzung der gesellschaftsvertragsartigen Zielbindung der Union (Förderung des Friedens, ihrer Werte und des Wohlergehens ihrer Völker), unabhängig von deren Neuformulierung im Einzelnen in Abweichung zu den derzeitigen Artt. 2-4 EGV und Art. 2 EUV. Sie folgt weitgehend, wiewohl nicht durchgängig dem VVE. ${ }^{20}$ Auch die Methode, die Zielverwirklichung an definierte Verwirklichungswege zu binden, bleibt trotz auffälliger Textneuerungen erhalten. Redaktionell mit eigenem Absatz als operativ erstgenannter Weg herausgestellt erscheint nunmehr allerdings die Schaffung eines Raums der Freiheit, der Sicherheit und des Rechts und erst sodann die Errichtung des Binnenmarktes, letzterer verbunden mit der Durchführung flankierender interner Politiken, gefolgt von der wie im derzeitigen Art. 2 EGV aufgeführten (aber vom VVE aus dessen Zielartikel verbannten) Errichtung einer Wirtschafts- und Währungsunion (mit zusätzlicher Akzentuierung ,,deren Währung der Euro ist“) und schließlich komplettiert durch die Verfolgung einer unionalen Politik ,,in ihren Beziehungen zur übrigen Welt.“21

Merkmale der Hoheitsstruktur: Die konzeptionelle Kontinuität manifestiert sich drittens auch in der Beibehaltung von drei charakteristischen Verbandsmerkmalen in der Gestaltung der Hoheitsstruktur. Zum einen wird die kombinative Natur des derzeitigen Unionsgedankens fortgeführt, die sowohl supranationale als auch intergouvernementale Elemente des Handelns umschließt. Des Weiteren wird der zwar nicht bundesstaatliche, wohl aber föderationsähnliche Charakter ${ }^{22}$ bestätigt. Zwar wird der (seit bald 45 Jahren vom EuGH ausgesprochene ${ }^{23}$ ) Anwendungsvorrang des supranationalen Primärrechts gegenüber abweichendem mitgliedstaatlichen Recht anders als vom VVE ${ }^{24}$ nicht mehr ausdrücklich im Vertragstext aufgeführt, sondern dessen Existenz nur in der anhängenden, nicht primärrechtlichen 17. Erklärung (unter Beifügung des Gutachtens des Juristischen Dienstes des

17 Der projektierte neue Art. 48 Abs. 6 UAbs. 2 EUV macht das Inkrafttreten des Beschlusses des Europäischen Rates (bezüglich Änderungen von Bestimmungen des Dritten Teils des AV) von der Zustimmung der Mitgliedstaaten im Einklang mit ihren jeweiligen verfassungsrechtlichen Vorschriften abhängig.

18 Der projektierte neue Art. 48 Abs. 7 UAbs. 3 EUV setzt für eine Beschlussfassung des Europäischen Rates (bezüglich des Übergangs vom Einstimmigkeitserfordernis zur Entscheidungsmöglichkeit mit qualifizierter Mehrheit oder des Übergangs von einem besonderen Gesetzgebungsverfahren beim Erlass von Gesetzgebungsakten durch den Rat zum ordentlichen Gesetzgebungsverfahren) voraus, dass keines der nationalen Parlamente innerhalb von sechs Monaten nach Übermittlung der Initiative des Europäischen Rates diese abgelehnt hat.

19 Art. 1 Ziff. 58 VvL: Art. 49a EUV = projektierter neuer Art. 50 EUV; vgl. dazu Art. I-60 VVE.

20 Art. 1 Ziff. 4 VvL: Art. 2 EUV = projektierter neuer Art. 3 EUV; vgl. dazu Art. I-3 VVE; zu den Abweichungen im Verhältnis zum VVE im Einzelnen vgl. unten im Text.

$21 \mathrm{Zu}$ diesen Bestimmungen im VVE vgl. Peter-Christian Müller-Graff: Die primärrechtlichen Grundlagen der Außenbeziehungen der Europäischen Union, in: Peter-Christian Müller-Graff (Hrsg.): Die Rolle der erweiterten Europäischen Union in der Welt, Baden-Baden 2006, S. 11-24.

22 Zu Einzelementen vgl. Peter-Christian Müller-Graff: The German Länder: Involvement in EC/EU Law and Policy Making, in: Stephen Weatherill/Ulf Bernitz (Hrsg.): The Role of Regions and Sub-national Actors in Europe, Oxford 2005, S. 103-118, hier S. 103, 105.

23 Grundlegend EuGH Slg. 1964, 1251 (Costa/ENEL).

24 Dort Art. I-6 VVE. 
Rates zum Vorrang vom 22. Juni 200725) in Erinnerung gerufen. Doch stärkt insbesondere der aus dem VVE übernommene, wiewohl nunmehr auseinandergerissene, systematische Abschnitt über die Kompetenzordnung ${ }^{26}$ das Pulsieren föderationsartiger, der Bundesverfassung des Grundgesetzes ähnelnder Kategorien. Gleiches gilt für den gleichfalls aus dem VVE übernommenen Grundsatz der wechselseitig loyalen Zusammenarbeit ${ }^{27}$ im Sinne einer allseitigen Unionstreue. ${ }^{28}$ Schließlich billigt der VvL ebensowenig wie der VVE der Union Exekutivgewalt in dem Sinne zu, dass diese eigenständig im Gebiet eines Mitgliedstaats Maßnahmen gegenüber Widerstrebenden unter Anwendung von Gewalt durchsetzen könnte.

\section{Steigerung des Potenzials der inneren und äußeren Handlungsfähigkeit}

Der erste große Teil der inhaltlich profilprägenden Neuerungen ist auf die Steigerung des Potenzials der inneren und äußeren Handlungsfähigkeit der Union gerichtet. Dem dienen insbesondere die Ergänzung der Kompetenzordnung, der neue Zuschnitt der Gesamtorganisation und die Neuerungen im Organgefüge.

Ergänzung der Kompetenzordnung: Die Kompetenzordnung erhält nicht nur das schon vom VVE vorgesehene textlich klarere Profil von allgemeinen Kompetenzprinzipien, Kompetenzkategorien und kategorialer Kompetenzklassifikation. ${ }^{29}$ Es wird zur Steigerung des Potenzials unionaler Handlungsfähigkeit auch um neue Verbandskompetenzen angereichert. Dies geschieht zum einen durch ausdrückliche Zuweisung neuer Handlungsfelder, zum anderen durch die Neufassung der Gesamtorganisation. Zu den textlich neu aufgenommenen Handlungsfeldern zählen im Einklang mit dem VVE namentlich Energie, ${ }^{30}$ Tourismus $^{31}$ und Katastrophenschutz ${ }^{32}$ sowie eine Reihe punktueller Handlungserweiterungen oder -klärungen (vor allem in den Bereichen des Schutzes geistiger Eigentumsrechte, ${ }^{33}$ der Raumfahrt, ${ }^{34}$ der Verwaltungszusammenarbeit, ${ }^{35}$ der Gemeinsamen Handelspolitik, ${ }^{36}$ der humanitären Hilfe $^{37}$ und der internationalen Übereinkünfte ${ }^{38}$ ) sowie über den VVE hinaus die Bekämpfung des Klimawandels ${ }^{39}$ und die Energieversorgung..$^{40}$ Bemerkenswert, aber zugleich problembegründend im Lichte der Handhabung des Subsidiaritätsprinzips, ist die auch schon

25 Dok. 11197/07: „Nach der Rechtsprechung des Gerichtshofs ist der Vorrang des EG-Rechts einer der Grundpfeiler des Gemeinschaftsrechts. Dem Gerichtshof zufolge ergibt sich dieser Grundsatz aus der Besonderheit der Europäischen Gemeinschaft. Zum Zeitpunkt des ersten Urteils im Rahmen dieser ständigen Rechtsprechung (Rechtssache 6/64, Costa gegen ENEL, 15. Juli 1964) war dieser Vorrang im Vertrag nicht erwähnt. Dies ist auch heute noch der Fall. Die Tatsache, daß der Grundsatz dieses Vorrangs nicht in den künftigen Vertrag aufgenommen wird, ändert nichts an seiner Existenz und an der bestehenden Rechtsprechung des Gerichtshofs."

26 Vgl. Art. 2 Ziff. 11 VvL: Artt. 2a-2e AV = projektierte neue Artt. 2-6 AV (vgl. dazu Artt. I-12 bis I-17 VVE); sowie Art. 1 Ziff. 6 VvL: Art. 3b EUV = projektierter neuer Art. 5 EUV (vgl. dazu Art. I-11 VVE).

27 Art. 1 Ziff. 5 VvL: Art. 3a EUV = projektierter neuer Art. 4 EUV; vgl. dazu Art. I-5 VVE.

28 Vgl. im deutschen Bundesverfassungsrecht den Gedanken der Bundestreue; zu dieser Michael Sachs: in Michael Sachs (Hrsg.): Grundgesetz-Kommentar, 3. Aufl., München 2003, Art. 20 Rdz. 68ff.

29 Siehe oben.

30 Art. 2 Ziff. 147 VvL: Art. 176a AV = projektierter neuer Art. 194 AV; vgl. dazu Art. III-256 VVE.

31 Art. 2 Ziff. 148 VvL: Art. 176b AV = projektierter neuer Art. 195 AV; vgl. dazu Art. III-281 VVE.

32 Art. 2 Ziff. 149 VvL: Art. 176c AV = projektierter neuer Art. 196 AV; vgl. dazu Art. III-284 VVE.

33 Art. 2 Ziff. 84 VvL: Art. 97a AV = projektierter neuer Art. 118 AV; vgl. dazu Art. III-176 VVE.

34 Art. 2 Ziff. 142 VvL: Art. 172a AV = projektierter neuer Art. 189 AV; vgl. dazu Art. III-254 VVE.

35 Art. 2 Ziff. 150 VvL: Art. 176d AV = projektierter neuer Art. 197 AV; vgl. dazu Art. III-285 VVE.

36 Art. 2 Ziff. 158 VvL: Art. 188c AV = projektierter neuer Art. 207 AV; vgl. dazu Art. III-315 VVE.

37 Art. 2 Ziff. 168 VvL: Art. 188j AV = projektierter neuer Art. 214 AV; vgl. dazu Art. III-321 VVE.

38 Art. 2 Ziff. 170-174 VvL: Artt. 1881-188o AV = projektierte neue Artt. 216-219 AV; vgl. dazu Artt. III-323, III-325 VVE.

39 Art. 2 Ziff. 143 VvL: Art. 174 Abs. 1 AV = projektierter neuer Art. 191 Abs. 1 AV.

40 Art. 2 Ziff. 87 VvL: Art. $100 \mathrm{AV}=$ projektierter neuer Art. $122 \mathrm{AV}$. 
vom VVE vorgesehene verbandskompetentielle Weitung des Aktionsradius in den Bereichen Visa, Einwanderung, Asyl und ziviljustitielle Zusammenarbeit infolge ihrer Entkoppelung von dem Erfordernis, dem reibungslosen Funktionieren des Binnenmarktes zu dienen. ${ }^{41}$

Neuer Zuschnitt der Gesamtorganisation: Neue Verbandskompetenzen ergeben sich aber auch infolge des neuen Zuschnitts der Gesamtorganisation. Diese erhält ihre neue Form durch die Verschmelzung von Europäischer Gemeinschaft und Europäischer Union. Unter dem Gesichtspunkt der Steigerung des Potenzials der Handlungsfähigkeit hervorhebenswert ist hierbei die Schaffung einer ungeteilten Rechtspersönlichkeit. ${ }^{42}$ Sie bedeutet, wie schon vom VVE vorgesehen, eine Ausweitung der europäischen Ebene als rechtlich eigenständiges Handlungs- und Zuordnungssubjekt für die gesamte Bandbreite der Verbandskompetenzen, hierin erstmals auch für die polizeiliche und justitielle Zusammenarbeit in Strafsachen ${ }^{43}$ und für die Außen- und Sicherheitspolitik. ${ }^{44}$ Im Bereich der polizeilichen und strafjustitiellen Zusammenarbeit kommt es zugleich zu dem schon vom VVE projektierten substanziellen Aufwuchs der Handlungsfelder namentlich durch die Ermächtigungen der Union, eine Europäische Staatsanwaltschaft einzurichten, ${ }^{45}$ Europol gebundene operative Maßnahmen durchführen zu lassen ${ }^{46}$ und Mindestvorschriften zur Festlegung von Straftaten und Strafen in Bereichen besonders schwerer Kriminalität mit grenzüberschreitender Dimension zu erlassen. ${ }^{47}$

Neuerungen im Organgefüge: Besonders herausragend und vielfältig sind die Neuerungen des VvL für das Organgefüge. Auch sie entsprechen im Wesentlichen denjenigen des VVE. Unter dem Gesichtspunkt der Steigerung der unionalen Handlungsfähigkeit betreffen sie namentlich: das Europäische Parlament durch die Festsetzung der Obergrenze seiner Mitgliederzahl auf 750; ${ }^{48}$ den Europäischen Rat durch die Einführung eines längerzeitigen Präsidentenamtes zur Koordinierung der (nunmehr) 27 Staats- und Regierungschefs der Mitgliedstaaten und zur Außenvertretung der Union in Angelegenheiten der Gemeinsamen Außen- und Sicherheitspolitik ,,auf seiner Ebene“ (protokollarisch zu verstehen), unbeschadet der Befugnisse des Hohen Vertreters der Union für Außen- und Sicherheitspolitik; ${ }^{49}$ den Ministerrat durch teilweises weiteres Abschmelzen des Beschlusserfordernisses der Einstimmigkeit zugunsten der Möglichkeit qualifizierter Mehrheitsentscheidungen ${ }^{50}$ (wobei die perspektivisch ab 1. November 2014 vorgesehene Neudefinition der qualifizierten Mehrheit

41 Vgl. zu dem darin schon dem VVE inhärenten Problem der Abgrenzung der Kompetenzausübung Peter-Christian Müller-Graff: Die ziviljustitielle Zusammenarbeit im „Raum der Freiheit, der Sicherheit und des Rechts“ im System des Europäischen Verfassungsvertrages, in: Heinz-Peter Mansel/Thomas Pfeiffer/Herbert Kronke/ Christian Kohler/Rainer Hausmann (Hrsg.): Festschrift für Erik Jayme, München 2004, S. 1323-1338, hier S. 1323, 1334f.; Peter-Christian Müller-Graff: Der „Raum der Freiheit, der Sicherheit und des Rechts“ im neuen Verfassungsvertrag - Neuerungen und Notwendigkeit seiner Rekonstruktion, in: Charlotte Gaitanides/Stefan Kadelbach/Gil Carlos Rodriguez Iglesias (Hrsg.): Europa und seine Verfassung. Festschrift für Manfred Zuleeg, Baden-Baden 2005, S. 605-623, hier S. 605ff., 613ff.

42 Art. 2 Ziff. 6 VvL.

43 Art. 2 Ziff. 67 und 69 VvL: Artt. 69a-69h AV = projektierte neue Artt. 82-89 AV; vgl. dazu Art. III-270-Art. III-277 VVE.

44 Art. 1 Ziff. 26-35, 37-40, 43-45, 47, 49-50 VvL: Artt. 10c-16, 18-21, 24-25b, 28-28e EUV = projektierte neue Artt. 23-46 EUV; vgl. dazu Art. III-294-Art. III-306, Art. III-308-Art. III-313; Art. I-40 Abs. 5, Art. I-41 VVE.

45 Art. 2 Ziff. 67 VvL: Art. 69e AV = projektierter neuer Art. 86 AV; vgl. dazu Art. III-274 VVE.

46 Art. 2 Ziff. 68 VvL: Art. 69g Abs. 3 AV = projektierter neuer Art. 88 Abs. 3 AV; vgl. dazu Art. III-276 VVE.

47 Art. 2 Ziff. 67 VvL: Art. 69b AV = projektierter neuer Art. 83 AV; vgl. dazu Art. III-271 VVE.

48 Art. 1 Ziff. 15 VvL: Art. 9a EUV = projektierter neuer Art. 14 EUV; vgl. dazu Art. I-20 VVE.

49 Art. 1 Ziff. 16 VvL: Art. 9b Abs. 5 EUV = projektierter neuer Art. 15 Abs. 5 EUV; vgl. dazu Art. I-22 VVE.

50 Eine nach dem Beschlusserfordernis aufgeschlüsselte Statistik zum VvL liegt noch nicht vor; als derartige Statistik für den VVE Andreas Maurer: Der Vertrag über eine Verfassung für Europa. Die neuen Handlungsermächtigungen der Organe. SWP-Diskussionspapier FG 1, März 2005, Tabellen 1 und 2, S. 19-20 sowie Abbildung 2, S. 39, wobei allerdings eine Bewertung dieser Entwicklung allein aufgrund einer (naturgemäß nicht nach Bedeutung wichtenden) statistischen Grundlage kaum möglich ist. 
als doppelte Mehrheit ${ }^{51}$ unter Effizienzgesichtspunkten insgesamt eher als neutral einzuschätzen ist) unter gleichzeitiger Erhebung der qualifizierten Mehrheit zum Regelabstimmungsverfahren ${ }^{52}$ (unter Aufgabe des derzeitigen, faktisch bedeutungslosen Grundsatzes der einfachen Mehrheit ${ }^{53}$ ) und unter Ausrufung des Mitentscheidungsverfahrens ${ }^{54}$ zum ordentlichen Gesetzgebungsverfahren, ${ }^{55}$ wobei jedoch wie schon im VVE gewichtige Agenden mit Einstimmigkeitserfordernis bleiben (namentlich in den Bereichen der Steuerangleichung und der Gemeinsamen Außen- und Sicherheitspolitik); die Kommission durch die (perspektivisch ab 1. November 2014 vorgesehene) Verkleinerung ihrer Mitgliederzahl auf 2/3 der Zahl der Mitgliedstaaten, ${ }^{56}$ wobei allerdings offen ist, ob der dadurch erhoffte Effizienzgewinn nicht von einem Akzeptanzverlust der Kommission in denjenigen Mitgliedstaaten begleitet wird, aus denen kein Staatsangehöriger der Kommission angehört, und ob mit dieser, im Vergleich zu den anderen politischen Organen asymmetrischen Repräsentanzlösung eine Diskursschwächung der Kommission eintritt: im Verhältnis insbesondere zum Europäischen Rat und (Minister-)Rat, die jeweils Vertreter aller Mitgliedstaaten umfassen, aber auch zu dem aus allen Mitgliedstaaten beschickten Europäischen Parlament; schließlich die Außenvertretung der Union durch die Schaffung des neuen Amtes eines Hohen Vertreters der Union für Außen- und Sicherheitspolitik mit der Doppelfunktion als Vorsitzender des Rates ,Auswärtige Angelegenheiten ' und als Kommissions-Vizepräsident ${ }^{57}$ sowie durch den Aufbau eines Europäischen Auswärtigen Dienstes ${ }^{58}$ und durch die Übertragung der Außenrepräsentanz der Union in Angelegenheiten der Gemeinsamen Außen- und Sicherheitspolitik auf den Präsidenten des Europäischen Rates. ${ }^{59}$

\section{Erhöhung der Legitimation unionaler Maßnahmen}

Der zweite große Teil der inhaltlich profilprägenden Neuerungen des VvL zielt, wie schon der VVE, auf die Stärkung der Legitimation unionaler Maßnahmen. Da kein hoheitliches Handeln selbstverständlich ist, sondern der Legitimation und in diesem Sinne seiner Verfasstheit bedarf, stellt sich dauerhaft die Frage nach deren funktions- und zeitgemäßer Ausgestaltung. Hierbei geht es sowohl um die sachgegenständliche als auch um die prozedural vermittelte Legitimation. Erstere versucht der VvL mit den sachgebietlichen Kompetenzerweiterungen $^{60}$ sowie der (wie im VVE nicht gänzlich gelungenen ${ }^{61}$ ) kategorialen

51 Art. 1 Ziff. 17 VvL: Art. 9c Abs. 4 EUV = projektierter neuer Art. 16 Abs. 4 EUV; vgl. dazu Art. I-25 VVE.

52 Art. 1 Ziff. 17 VvL: Art. 9c Abs. 3 EUV = projektierter neuer Art. 16 Abs. 3 EUV; vgl. dazu Art. I-23 Abs. 3 VVE.

53 Vgl. Art. 205 Abs. 1 EGV.

54 Vgl. Art. 251 EGV.

55 Art. 2 Ziff. 236 VvL: Art. 249a AV = projektierter neuer Art. 289 AV; vgl. dazu Art. I-34, Art. III-396 VVE.

56 Art. 1 Ziff. 18 VvL: Art. 9d Abs. 5 EUV = projektierter neuer Art. 17 Abs. 5 EUV; vgl. dazu Art. I-26 Abs. 6 VVE.

57 Art. 1 Ziff. 19 VvL: Art. 9e EUV = projektierter neuer Art. 18 EUV; vgl. dazu Art. I-28 VVE.

58 Art. 1 Ziff. 30 VvL: Art. 13a Abs. 3 EUV = projektierter neuer Art. 27 Abs. 3 EUV; vgl. dazu Art. III-296 Abs. 3 VVE.

59 Vgl. oben.

60 Vgl. oben.

61 Unerwähnt bleiben die spezifischen Mitwirkungszuständigkeiten unionaler Organe in der Primärrechtspolitik. Unklar bleibt die Zuordnung der binnenmarktintegrativen Rechtsangleichungszuständigkeit zur Überwindung von Wettbewerbsverzerrungen aus unterschiedlichen mitgliedstaatlichen Vorschriften zwischen der ausschließlichen Zuständigkeit zur „Festlegung der für das Funktionieren des Binnenmarkts erforderlichen Wettbewerbsregeln“ und der geteilten Zuständigkeit „Binnenmarkt“. Außerhalb des vom VVE geschaffenen Systems mit seiner vertragssystematischen Zuordnungsregel des Art. I-14 Abs. 1 zur geteilten Zuständigkeit bleibt nach dem VvL die aus den allgemeinen Kompetenzregeln des AV herausgenommene und in den EUV verfrachtete Gemeinsame Außen- und Sicherheitspolitik. 
Klassifizierung der einzelnen Sachkompetenzen ${ }^{62}$ sowie der konzisen Bündelung der Zielbindung $^{63}$ zu stärken. Letztere ist, gleichfalls in Rezeption des VVE, Gegenstand bemerkenswerter Neuerungen. ${ }^{64}$

Prozedurale Vorkehrungen: Auf die Erhöhung der prozedural vermittelten Legitimation zielen zahlreiche, überwiegend aus dem VVE übernommene, neue Vorkehrungen, darunter namentlich sachgegenständliche Ausweitungen des Mitentscheidungsverfahrens zwischen dem (demokratietheoretisch freilich weiterhin nicht modellgemäß zusammengesetzten) Europäischen Parlament und dem Rat unter gleichzeitiger Erhebung dieses Verfahrens zum „ordentlichen Gesetzgebungsverfahren“, 65 die Berücksichtigungspflicht der Ergebnisse der Wahlen zum Europäischen Parlament beim Vorschlag eines Kandidaten für das Amt des Kommissionspräsidenten durch den Europäischen Rat an das Europäische Parlament, ${ }^{66}$ die Stärkung des Europäischen Parlaments bei der Nutzung der Abrundungsermächtigung des Art. 308 AV (,Zustimmung“), die Öffentlichkeitspflicht der Tagungen des Rates bei Beratungen oder Abstimmungen über Entwürfe zu Gesetzgebungsakten ${ }^{67}$ sowie die systematische Bündelung und Hervorhebung der drei Grundsätze der demokratischen Gleichheit, der repräsentativen Demokratie und der öffentlich-partizipativen Demokratie in einem eigenen Titel. ${ }^{68}$ Als legitimationssteigernd lässt sich auch die ausdrückliche normtextliche Grundrechtsbindung der Unionsorgane durch Aufnahme der Grundrechtecharta in das Primärrecht verstehen, auch wenn sie ohne die vom VVE vorgesehene Textinkorporation nunmehr allein mittels des Satzes erfolgt: „die Charta der Grundrechte und die Verträge sind rechtlich gleichrangig"“ ${ }^{69}$ Diese Verweistechnik ist angesichts der Üppigkeit und rechtlich konglomeraten Struktur, ${ }^{70}$ aber auch angesichts der Vorbehalte Britanniens und Polens gegen ihre Erstreckungswirkung auf nationale Maßnahmen ${ }^{71}$ textsystematisch durchaus gut vertretbar.

Neudefinition der qualifizierten Mehrheit als doppelte Mehrheit: Besonders herausragend für die Legitimationserhöhung sind die Neudefinition der qualifizierten Mehrheit im Rat und die Einbeziehung der nationalen Parlamente. So ist der erneut nach zähen Verhandlungen ${ }^{72}$ erzielte Durchbruch zur systemrationalen Neudefinition der qualifizierten Mehrheit im Rat trotz aller komplizierten Begleitkompromisse und Einschränkungen ${ }^{73}$ ein legitimatorisch epochaler Schritt. Wie schon im VVE vorgesehen gilt dadurch als qualifizierte Mehr-

62 Scil.: ausschließliche, geteilte, fördernde; vgl. oben.

63 Vgl. oben.

64 Vgl. unten

65 Vgl. oben

66 Art. 1 Ziff. 18 VvL: Art. 9d Abs. 7 S. 1 EUV = projektierter neuer Art. 17 Abs. 7 S. 1 EUV; vgl. dazu Art. I-27 Abs. 1 VVE.

67 Art. 1 Ziff. 17 VvL: Art. 9c Abs. 8 EUV = projektierter neuer Art. 16 Abs. 8 EUV; vgl. dazu Art. I-24 Abs. 6 VVE.

68 Art. 1 Ziff. 12 VvL: Artt. 8-8c EUV = projektierte neue Artt. 9-12 EUV; vgl. dazu Art. I-45-Art. I-47 VVE.

69 Art. 1 Ziff. 8 VvL: Art. 6 Abs. 1 EUV = projektierter neuer Art. 6 Abs. 1 EUV; im VVE figurierte die Charta als umfänglicher Zweiter Teil des Gesamttextes.

70 Vgl. als neueste kritische Würdigungen der Charta Rudolf Streinz: Wie gut ist die Grundrechte-Charta des Verfassungsvertrages?, in: Jörn Ipsen/Bernhard Stüer (Hrsg.): Europa im Wandel. Festschrift für Hans-Werner Rengeling, München 2008, S. 645, 657ff.; Torsten Stein: Haben die Engländer nicht eigentlich recht? Bemerkungen zu einer verpassten Chance für die Europäische Grundrechtecharta, in: Zeitschrift für Europarecht (EuZ) 2/2008, S. 37-43.

71 Protokoll über die Anwendung der Charta der Grundrechte der Europäischen Union auf Polen und das Vereinigte Königreich.

72 Vgl. dazu Fischer: Der Vertrag von Lissabon, 2008, S. 71ff., 75ff.; speziell zur ,Quadratwurzel‘-Debatte vgl. Stefan Tomik: Quadratwurzel-Behandlung für Europa, in: Frankfurter Allgemeine Zeitung, 14.06.2007; dazu Müller-Graff: Die Zukunft des europäischen Verfassungstopos, 2007, S. 231.

73 Dies gilt namentlich für das Hinauszögern des Inkrafttretens auf den 1. November 2014, die Übergangsbestimmungen bis zum 31. März 2017 und die Sperrminoritätenregel; vgl. Art. 1 Ziff. 17 VvL: Art. 9c Abs. 4 und 5 $\mathrm{EUV}=$ projektierter neuer Art. 16 Abs. 4 und 5 EUV und das Protokoll über die Übergangsbestimmungen. 
heit eine Mehrheit von 55 Prozent der Mitglieder des Rates, gebildet aus mindestens 15 Mitgliedern, sofern die von diesen vertretenen Mitgliedstaaten zusammen mindestens 65 Prozent der Bevölkerung der Union ausmachen.$^{74}$ Eine derartige Bewertung ist naturgemäß abhängig vom Verständnis der Wesensnatur der Union und oft genug erörtert, ${ }^{75}$ sodass sie in dieser Skizze der wesentlichen Neuerungen nur einer kurzen Erinnerung bedarf. Versteht man die Union als transnationales Gemeinwesen, ${ }^{76}$ ist es folgerichtig, eine Doppellegitimation der Wahrnehmung ihrer Befugnisse insbesondere auch im Legislativbereich zu fordern: eine völkerrechtstheoretisch überzeugende Legitimation seitens der vom Gemeinschaftshandeln betroffenen Mitgliedstaaten und eine demokratietheoretisch sinnfällige Legitimation seitens der betroffenen Unionsbürger. Beides wird über das Europäische Parlament weder derzeit noch nach den Neuerungen des VvL hinreichend vermittelt: eine völkerrechtstheoretisch überzeugende Legitimation nicht, weil die Mitglieder des Parlaments nicht Vertreter der Mitgliedstaaten, sondern in der - aus dem VVE übernommenen - Neuformulierung des VvL ,der Unionsbürgerinnen und Unionsbürger"77 sind; eine demokratietheoretisch überzeugende Legitimation nicht hinreichend, weil trotz der Direktwahl der Mitglieder, wie bekannt, bereits die Zusammensetzung des Parlaments nach dem Vertretungsprinzip der degressiven Proportionalität der mitgliedstaatlichen Unionsbürgerschaften die Unionsbürger nicht nach dem Grundsatz deren Gleichwertigkeit (also nicht nach dem Prinzip der linearen Proportionalität) abbildet. Angesichts dieser konzeptionellen Legitimationsvermittlungsschwäche des Parlaments ist es folgerichtig, diese über das Entscheidungsverfahren im Rat zu kompensieren, der sich aus je einem Vertreter jedes Mitgliedstaats zusammensetzt, ${ }^{78}$ wobei jeder Mitgliedstaat (wie schon derzeit ${ }^{79}$ ) auf den ,Wert ${ }^{\star}$ der Demokratie verpflichtet ist. ${ }^{80}$ Soweit im Rat das Verfahren der qualifizierten Mehrheitsentscheidung zur Anwendung kommt, folgt jedoch die derzeitige Definition der qualifizierten Mehrheit mit ihrer Stimmgewichtung der Mitgliedstaaten nach dem Prinzip der degressiven Proportionalität keiner legitimatorischen Systemrationalität. Diese gegenwärtige Schwäche wird vom VvL nunmehr mit dem Prinzip der doppelten Mehrheit zugunsten einer legitimationstheoretisch optimalen Kombinationslösung überwunden. Es erfüllt in seinem Kern gleichermaßen die legitimatorischen Erfordernisse der Gleichwertigkeit jedes Mitgliedstaats und jedes Unionsbürgers.

Einbau der nationalen Parlamente in die unionale Legitimationsarchitektur: Für die legitimatorische Stärkung nicht minder bedeutsam ist die vom VvL in eigenständiger Fortentwicklung des VVE nunmehr ausdrücklich in die Bestimmungen über die demokratischen Grundsätze gebündelt einbezogene und dadurch hervorgehobene Rolle der nationalen Parlamente in der Architektur der Union ${ }^{81}$ und deren aus dem VVE übernommene Verstärkung. Den nationalen Parlamenten wird generell die (nicht einklagbare) Aufgabe zugewiesen, ,,aktiv zur guten Arbeitsweise der Union (beizutragen)“, und diese Aufgabe sodann in sechsfacher Ausfaltung aufgefächert. Die neue Norm verdeutlicht einen (vorsichtigen) Rückgriff der Union auf das Legitimationspotenzial nationaler Parlamente. $\mathrm{Zu}$ den schon bestehenden Unterrichtungsrechten gegenüber den Organen der Union und Beteiligungsregeln der interparlamentarischen Zusammenarbeit kommen nunmehr differenziert ausgestaltete Einbezie-

74 Art. 1 Ziff. 17 VvL: Art. 9c Abs. 4 EUV = projektierter neuer Art. 16 EUV; vgl. Art. I-25 VVE.

75 Zur eigenen Position vgl. Müller-Graff: Strukturmerkmale des neuen Verfassungsvertrages, 2004, S. $197 f$.

76 Zur Begründung Peter-Christian Müller-Graff: Verfassungsziele der EU/EG, in: Manfred Dauses (Hrsg.): Handbuch des EU-Wirtschaftsrechts, München 2000, A I Rdz. 72ff.

77 Art. 1 Ziff. 15 VvL: Art. 9a Abs. 2 EUV = projektierter neuer Art. 14 Abs. 2 EUV; vgl. dazu Art. I-20 VVE.

78 Art. 1 Ziff. 17 VvL: Art. 9c Abs. 2 EUV = projektierter neuer Art. 16 Abs. 2 EUV; vgl. dazu Art. I-23 VVE.

79 Art. 6 Abs. 1 EUV.

80 Art. 1 Ziff. 3 VvL: Art. 1a S. 2 EUV = projektierter neuer Art. 2 S. 2 EUV; vgl. dazu Art. I-2 VVE.

81 Art. 1 Ziff. 12 VvL: Art. 8c EUV = projektierter neuer Art. 12 EUV; ohne textliche Entsprechung im VVE. 
hungen in sensiblen Fragen hinzu. So wird insbesondere in Übernahme des einschlägigen Protokolls zum VVE für die Anwendung des Subsidiaritätsgrundsatzes ein neuartiges politisches Frühwarnsystem zugunsten der nationalen Parlamente eingeführt. ${ }^{82}$ Es ist von der Lissabonner Fassung speziell im Rahmen des ordentlichen Gesetzgebungsverfahrens verschärft. ${ }^{83}$ Zugleich begründet das Protokoll die Zuständigkeit des EuGH auch für Nichtigkeitsklagen wegen Verstoßes gegen das Subsidiaritätsprinzip, die von einem Mitgliedstaat im Namen seines nationalen Parlaments oder einer Kammer dieses Parlaments übermittelt werden. Weitere Beteiligungen der nationalen Parlamente betreffen die Bewertung der Durchführung der Unionspolitiken im Rahmen des Raums der Freiheit, der Sicherheit und des Rechts (Beteiligung) ${ }^{84}$, die politische Kontrolle von Europol (Einbeziehung), ${ }^{85}$ die Bewertung der Tätigkeit von Eurojust (Einbeziehung), ${ }^{86}$ das zweite vereinfachte Vertragsänderungsverfahren (Ablehnungsrecht) ${ }^{87}$ und Beitrittsanträge (Unterrichtung). ${ }^{88}$

\section{Relativer Rückschnitt von Texttransparenz und Staatsanalogisierung im Vergleich zum VVE}

Ein markantes äußeres Kennzeichen des VvL im Vergleich zum VVE ist der relative Rückschnitt von Texttransparenz und Staatsanalogisierung, wiewohl beides nicht gänzlich aufgegeben wird und insoweit in der Änderungsbilanz zum derzeitigen Primärrecht durchaus Fortentwicklungen zu verzeichnen sind.

Zurückgenommene Transparenz: Das vom VVE zum Zweck der seit vielen Jahren geforderten Texttransparenz und Komplexitätsreduktion vorgelegte Angebot einer systematisch übersichtlich durchkomponierten Gesamtkodifikation des zentralen (also nicht in Protokollen ausgefalteten) Primärrechts wird vom vorliegenden Reformvertrag nicht mehr mit gleicher Entschiedenheit angestrebt noch in vergleichbarer Stringenz wie vom VVE geleistet. Allerdings ist dieses mit dem VVE verfolgte Ziel keineswegs völlig aus den Augen verloren worden, zumal es einem gereiften normativen Verständnis ohnehin nahezu unmöglich ist, hinter einen einmal erreichten Einsichts- und Systematisierungsstand zurückzufallen. Schon der makrooptischen Betrachtung erschließt sich rasch, dass insbesondere die Verschmelzung von Gemeinschaft und Union zu einer einheitlichen Organisation einen nicht zu unterschätzenden Transparenzgewinn für die Öffentlichkeit ermöglicht. Gleiches gilt für den damit einhergehenden Wegfall der zweigeteilten Lokation und Rechtsnatur der Bestimmungen über den sogenannten Raum der Freiheit, der Sicherheit und des Rechts in $\mathrm{EGV}^{89}$ und $\mathrm{EUV}^{90}$ zugunsten dessen aus dem VVE übernommenen, systematisch kohärent aufgebauten Zusammenfassung, wobei nunmehr ${ }^{91}$ auch textsystematisch der herkömm-

82 Art. 6 und 7 des Protokolls über die Anwendung der Grundsätze der Subsidiarität und der Verhältnismäßigkeit.

83 Ebenda: Art. 7 Abs. 3.

84 Art. 2 Ziff. 64 VvL: Art. 61c AV = projektierter neuer Art. 71 AV; vgl. dazu Art. III-261 VVE.

85 Art. 2 Ziff. 68 VvL: Art. 69g Abs. 2 AV = projektierter neuer Art. 88 Abs. 2 AV; vgl. dazu Art. III-276 VVE.

86 Art. 2 Ziff. 67 VvL: Art. 69d AV = projektierter neuer Art. 85 AV; vgl. dazu Art. III-273 VVE.

87 Art. 1 Ziff. 56 VvL: Art. 48 Abs. 7 EUV; vgl. dazu Art. III-444 VVE.

88 Art. 1 Ziff. 57 VvL: Art. 49 EUV = projektierter neuer Art. 49 EUV.

89 Artt. 61ff. EGV.

90 Artt. 29ff. EUV.

91 Anders die Lokation im VVE, in dem die Vorschriften über den Raum der Freiheit, der Sicherheit und des Rechts auf das Kapitel über die Wirtschafts- und Währungspolitik und dasjenige über die Politik ,,in anderen Bereichen" folgten: Artt. III-257ff. VVE. 
liche und normgenetisch konzeptionsstimmige ${ }^{92}$ Anschluss an die Binnenmarktvorschriften beibehalten wird. ${ }^{93}$ Ebenso transparenzerhöhend wirken dürfte der damit einhergehende Wegfall der für Laien anscheinend verwirrenden besonderen rechtlichen Maßnahmetypen in der derzeitigen dritten Säule (namentlich des Rahmenbeschlusses ${ }^{94}$ ) zugunsten der vertrauten Maßnahmetypen des derzeitigen EGV, ${ }^{95}$ von denen allerdings die ,Entscheidung “ terminologisch durch den ,Beschluss “ ersetzt wird. ${ }^{96}$ Auch die Zahl der verschiedenen Entscheidungsverfahren wird dadurch reduziert. Überdies wird, wie schon aufgewiesen, das Mitentscheidungsverfahren in den Rang des ,ordentlichen Gesetzgebungsverfahrens “ in Abgrenzung zu ,besonderen Gesetzgebungsverfahren“ erhoben. ${ }^{97}$ Aus der Systematik des VVE gerettet ist auch der profilbildende Teil der Organbestimmungen..$^{98}$

Der VvL schafft aber keinen einheitlichen Vertragstext, sondern agiert mit zwei (vorerst?99) ,gleichrangigen“ Verträgen sowie der vertragsgleich gestellten Grundrechte-Charta. Diese Trichotomie erinnert allerdings an die Dreiteilung des VVE in den profilbildenden Ersten Teil, den speziell den Grundrechten gewidmeten Zweiten Teil und den speziell den Politikbereichen und der Arbeitsweise der Union gewidmeten Dritten Teil. Jedoch folgt die Ausfüllung nicht komplett derjenigen des VVE, sondern produziert teils inhaltlich bizzar anmutende Trennungen. Letzteres gilt für die Lokation der grundlegenden Normen zur Kompetenzordnung. Der für den VVE entworfene einheitliche Abschnitt ist nunmehr in drei Teile auseinandergenommen. Die allgemeinen Kompetenzprinzipien (Prinzip der begrenzten Einzelermächtigung, Subsidiaritätsprinzip, Grundsatz der Verhältnismäßigkeit) finden sich im EUV als Art. 3b EUV ${ }^{100}$ wieder, ergänzt um einen vorlaufenden, vom VvL neu kreierten klarstellenden neuen Art. 3a Abs.1 EUV, ${ }^{101}$ dass alle der Union nicht in den Verträgen übertragenen Zuständigkeiten gemäß Art. 3b EUV bei den Mitgliedstaaten verbleiben. Die im VVE entwickelten allgemeinen Kompetenzkategorien und kategorial zugeordneten $\mathrm{Zu}-$ ständigkeitsbereiche sind (unter Herausnahme der damit in ihrer kompetentiellen Kategorialität wieder offenen Gemeinsamen Außen- und Sicherheitspolitik) im AV als Artt. 2a-2d ${ }^{102}$ platziert. Die Flexibilitätsklausel schließlich bleibt in Art. 308 AV. ${ }^{103}$ Auch die übersichtliche systematische Kodifikationsleistung der Bestimmungen über das auswärtige Handeln der Union durch den VVE ${ }^{104}$ wird zerlegt. Sie wird teils neu zusammengesetzt, teils in die derzeitige lokative Zergliederung überführt, doch wird der vom VVE erreichte Systematisierungsansatz letztlich in einer Zweiteilung gerettet. Die allgemeinen Bestim-

92 Die in Artt. 61ff. EGV aufgeführten Gegenstände waren zuvor als Gegenstände gemeinsamen Interesses in die dritte Unionssäule des Unionsvertrages von Maastricht aufgenommen, weil der angestrebte binnengrenzkontrollose Binnenmarkt entsprechende flankierende Maßnahmen erforderte; vgl. dazu Peter-Christian MüllerGraff: Die Europäische Zusammenarbeit in den Bereichen Justiz und Inneres, in: Ole Due/Marcus Lutter/Jürgen Schwarze (Hrsg.): Festschrift für Ulrich Everling, Baden-Baden 1995, S. 925-944, hier S. 925, 940.

93 Gemäß Art. 2 Ziff. 64-68 VvL: Artt. 61-69h AV = projektierte neue Artt. 67-89 AV.

94 Vgl. Art. 34 Abs. 2 lit.b EUV.

95 Vgl. Art. 249 EGV.

96 Art. 2 Ziff. 235 VvL: Art. 249 Abs. 4 AV = projektierter neuer Art. 288 Abs. 4 AV.

97 S. oben.

98 Art. 1 Ziff. 13 VvL: Artt. 9-9f. EUV = projektierte neue Artt. 13-19 EUV; vgl. dazu Artt. I-19-I-29 VVE.

99 Auffällig bleibt jedenfalls, dass die Bestimmungen des Dritten Teils des AV über die internen Politikbereiche nach dem VvL grundsätzlich dem vereinfachten Vertragsänderungsverfahren zugänglich sind; dies entspricht den Regelungen des VVE zu den Bestimmungen des Teils III Titel III über die internen Politikbereiche (vgl. Art. III-445 VVE).

100 Art. 1 Ziff. 6 VvL: Art. 3b EUV = projektierter neuer Art. 5 EUV.

101 Art. 1 Ziff. 5 VvL: Art. 3 a EUV = projektierter neuer Art. 4 EUV.

102 Art. 2 Ziff. 12 VvL: Artt. 2a-2e AV = projektierte neue Artt. 2-6 AV.

103 Art. 2 Ziff. 289 VvL: Art. 308 AV = projektierter neuer Art. 352 AV.

104 Vgl. Artt. III-292-III-329 VVE sowie Art. I-16, Art. I-40, Art. I-42 VVE. 
mungen und die besonderen Bestimmungen über die Gemeinsame Außen- und Sicherheitspolitik des VVE (Teil III Titel V Kapitel I und II sowie Teil I Titel V Kapitel II) ${ }^{105}$ treten unter teilweiser Umformulierung und Umschichtung im Vergleich zum VVE an den Normort der derzeitigen zweiten Unionssäule als umfängliche Artt. 10a-28e EUV. ${ }^{106}$ Die anderen Kapitel im Dritten Teil des VVE (Kapitel III-VIII), ${ }^{107}$ mithin die Bestimmungen über die Gemeinsame Handelspolitik, die Zusammenarbeit mit Drittländern und humanitäre Hilfe, die restriktiven Maßnahmen, die internationalen Übereinkünfte, die Beziehungen der Union zu internationalen Organisationen und Drittländern sowie Delegationen der Union und die Solidaritätsklausel sind in der nämlichen Reihung und unter lediglich geringfügigen textlichen und inhaltlichen Änderungen zum VVE zu einem neuen eigenen Teil des AV unter der parallelen Überschrift „Das Auswärtige Handeln der Union“ ausgeformt. ${ }^{108}$

Zurückgenommene Staatsanalogisierung: In der Öffentlichkeit wurde als augenfälligster Unterschied des VvL zum VVE der Verzicht auf die staatsanalogisierenden Attribute des VVE wahrgenommen. Er wird zuallererst in der Aufgabe der Gesamtkodifikation in einem einzigen Vertrag mit der Bezeichnung, Vertrag über eine Verfassung für Europa ‘ ersichtlich sowie in der durchgängigen Ersetzung des ohnehin eher mißverständlichen Kurzbegriffs ,Verfassung' zur Selbstbezeichnung des Primärrechts durch den Begriff ,Verträge‘. Man kann füglich der Ansicht sein, dass sich für die kategoriale Qualität des Primärrechts weder durch die Bezeichnung als, Verfassung ' noch durch den nunmehrigen Verzicht darauf etwas ändert. Auch der VVE war ein Vertrag, wie sich schon aus seinem Titel und den Artt. IV437-IV-448 VVE ergab. Und auch der Lissabonner Reformvertrag beinhaltet nicht anders als der derzeitige EGV Bestimmungen zur Legitimation und Kontrolle der europäischen Hoheitsordnung ${ }^{109}$ und steht damit in der Tradition des EWGV, den das Bundesverfassungsgericht ,gewissermaßen die Verfassung dieser Gemeinschaft“" nannte. ${ }^{110}$ Aus dieser rechtlichen Sicht wirkt die Diskussion um die Angemessenheit oder Zulässigkeit des Verfassungsvertragsbegriffs für die europäische Ebene als terminologische Oberflächendebatte, der allerdings ersichtlich gewaltige Sprengkraft im Freilandversuch der öffentlichen Meinungen und politischen Praxis innewohnt. Zur Wahrnehmung der etatistischen Entanalogisierung des VvL trägt insbesondere auch dessen Verzicht auf die Übernahme des Symbolartikels des VVE 111 bei, der Flagge, Hymne, Leitspruch, Währung und Europatag festschrieb. Freilich wird nahezu nichts von alledem aus der teils langjährigen Praxis verschwinden. Ob die Streichung der symbolhaft exponierten textlichen Proklamation „In Vielfalt geeint" vielfaltsbeeinträchtigende Folgen zeitigt, ist spekulativ. Auch die Nichtübernahme des ausdrücklichen Ausspruchs des Vorrangs unionalen Rechts vor mitgliedstaatlichem Recht ${ }^{112}$ beinhaltet, wie schon gezeigt, keine Änderung des seit mehr als vier Jahrzehnten vom EuGH judizierten ${ }^{113}$ Anwendungsvorrangs des dazu geeigneten europäischen Rechts. In die terminologische Abrüstung fügt sich auch die Abstandnahme des VvL von der im VVE enthaltenen, inhaltlich durchaus passfähigen Umbenennung von Verord-

105 Vgl. Artt. III-292-III-313 VVE sowie Art. I-16, Art. I-40, Art. I-42 VVE.

106 Projektierte neue Artt. 21-46 EUV; vgl. Art. 1 Ziff. 40-50 VvL.

107 Artt. III-314-III-329 VVE.

108 Art. 2 Ziff. 155-176 VvL: Artt. 188a-188r AV = projektierte neue Artt. 205-222 AV.

109 Vgl. Peter-Christian Müller-Graff: Europäische Verfassungsordnung - Notwendigkeit, Gestalt und Fortentwicklung, in: Europäisches Wirtschafts- und Steuerrecht (EWS) 5/2002, S. 206-216, hier S. 206, $210 f f$.

110 BVerfGE 22, 293 - EWG-Verordnungen.

111 Art. I-8 VVE.

112 Art. I-6 VVE.

$113 \mathrm{Vgl}$. oben. 
nung und Richtlinie in Europäisches Gesetz und Europäisches Rahmengesetz. ${ }^{114}$ Indes wird bei mikrooptischer Betrachtung des VvL der Gesetzesbegriff auf unionaler Ebene nur auf dieser plakativen Sichtebene vermieden, da er im prozeduralen Innenleben der Union im Begriff des „Gesetzgebers“, 115 des „Gesetzgebungsaktes“116 und des ordentlichen und besonderen „Gesetzgebungsverfahrens" 117 in das Primärrecht eingeführt wird.

Bemerkenswerterweise wird aber auch die neue Bezeichnung des VVE für den Legitimationsquell der Mitglieder des Europäischen Parlaments übernommen, der sich nicht mehr, wie derzeit, aus der Vertretung der Völker der in der Gemeinschaft zusammengeschlossenen Staaten ${ }^{118}$ speist, sondern aus der Vertretung der Unionsbürgerinnen und Unionsbürger. ${ }^{119}$ Deren Repräsentanz wird aber dann doch nach degressiv proportional zur Bevölkerungszahl bestimmten mitgliedstaatlichen Kontingenten parzelliert. ${ }^{120}$ Auch der schon vom VVE vorgesehene Ausbau der Regeln zur Verflechtung einzelner Sachpolitiken ${ }^{121}$ spiegelt die gemeinwesenartige Verdichtung der Union wider.

Man mag dem VvL als Paradoxie zu seiner Vorgeschichte sogar leichte Ansätze zu einem über den VVE hinausgehenden staatsanalogisierenden Denken entnehmen: so namentlich in der forcierten Vorziehung ${ }^{122}$ der demokratischen Grundsätze vor die Organbestimmungen und in dem nunmehr zum primären operativen Hauptziel in einem eigenen Absatz des Zielartikels noch vor dem Binnenmarkt erhobenen sogenannten Raum der Freiheit, der Sicherheit und des Rechts. ${ }^{123}$ Denn damit wird (vielleicht eher unwillentlich) auf den befriedeten freien Bewegungsraum angespielt, der an der Wiege der Entstehung moderner Staatlichkeit durch Schaffung des Gewaltmonopols stand. Der Union fehlt freilich auch mit der Lissabonner Reform jegliche derartige Macht.

\section{Konzeptionelle Komplexierung mit Einbußen an konzeptioneller Klarheit im operati- ven Primärrecht}

Bereits das Vorziehen des Raums der Freiheit, der Sicherheit und des Rechts lässt ahnen, dass der Reformvertrag mit Einbußen an konzeptioneller Klarheit im Vergleich zum operativen primärrechtlichen Acquis verbunden ist.

Neue Rolle des Raums der Freiheit, der Sicherheit und des Rechts? Das operative Zielkonzept des Raums der Freiheit, der Sicherheit und des Rechts weist keine räumlich, konzeptionell, inhaltlich und rechtlich vergleichbare Konturenklarheit auf ${ }^{124}$ wie der derzeitige primäre supranationale Verwirklichungsweg der Leitziele des EGV, nämlich die Errichtung eines Ge-

114 Vgl. Art. I-33 VVE.

115 Vgl. Art. 1 Ziff. 15 VvL: Art. 9a Abs. 1 EUV = projektierter neuer Art. 14 Abs. 1 EUV; Art. 1 Ziff. 17 VvL: Art. 9c Abs. 1 EUV = projektierter neuer Art. 16 Abs. 1 EUV.

116 Vgl. Art. 1 Ziff. 56 VvL: Art. 48 Abs. 7 UAbs. 2 EUV = projektierter neuer Art. 48 Abs. 7 UAbs. 2 EUV.

117 Vgl. z.B. Art. 2 Ziff. 236 VvL: Art. 249a Abs. 1 AV = projektierter neuer Art. 289 AV; Art. 48 Abs. 7 UAbs. 2 EUV.

118 Vgl. Art. 189 Abs. 1 EGV.

119 Art. 1 Ziff. 15 VvL: Art. 9a Abs. 2 EUV = projektierter neuer Art. 14 Abs. 2 EUV.

120 Art. 1 Ziff. 15 VvL: Art. 9a Abs. 2 S. 3 EUV = projektierter neuer Art. 14 Abs. 2 S. 3 EUV.

121 Art. 2 Ziff. 13ff. VvL: Artt. 2f-6b AV = projektierte neue Artt. 7-13 AV.

122 Vgl. einerseits Art. 1 Ziff. 12, 13-20 VvL: Artt. 8-8c EUV = projektierte neue Artt. 9-12 EUV und Artt. 9-9f EUV (= projektierte neue Artt. 13-19 EUV) und andererseits Art. I-19-Art. I-32 VVE und Art. I-45-Art. I-52 VVE.

123 Vgl. einerseits Art. 1 Ziff. 4 VvL: Art. 2 Abs. 2 EUV = projektierter neuer Art. 3 Abs. 2 EUV, andererseits Art. I-3 Abs. 2 VVE.

124 Zur konzeptionellen Offenheit in EGV/EUV im Einzelnen Peter-Christian Müller-Graff: Der Raum der Freiheit, der Sicherheit und des Rechts - Der primärrechtliche Rahmen, in: Peter-Christian Müller-Graff (Hrsg.): Der Raum der Freiheit, der Sicherheit und des Rechts, Baden-Baden 2005, S. 11-28; zum VVE: Art. 2 Ziff. 150 VvL: Art. 176d AV = projektierter neuer Art. 197 AV; vgl. dazu Art. III-285 VVE. 
meinsamen Marktes. ${ }^{125}$ Nehmen an diesem grundsätzlich alle Mitglieder teil, so gilt dies nicht in gleicher Weise für den , allgemeinen 'Freiheitsraum, zu dem in unterschiedlicher Weise Besonderheiten für Britannien, Irland und Dänemark bestehen. ${ }^{126}$ Fußt der Gemeinsame Markt (beziehungsweise der Binnenmarkt) auf dem theoretischen Konzept des (schutzgutumrahmten) wettbewerbseröffnenden Prinzips des komparativen Kostenvorteils, ${ }^{127}$ so bleibt die Gestalt des Raums der Freiheit, der Sicherheit und des Rechts inhaltlich heterogen, ohne einheitliche theoretische Fundierung und auch nicht sinnfällig ohne den Sockel des binnenmarktlichen Freiheitsraums. ${ }^{128}$ Und wird der Binnenmarkt von primärrechtlich gewährleisteten, unmittelbar anwendbaren Grundfreiheiten konstituiert, ${ }^{129}$ so beruht der allgemeine Freiheitsraum primärrechtlich allein auf Ermächtigungsnormen, deren Nutzung von der politischen Diskretion der Mitgliedstaaten abhängt. Eine Ausnahme bildet nur die unionsbürgerliche Gewährleistung des freien Personenverkehrs, die zwar textsystematisch nicht dem einschlägigen Raum-Kapitel zugehört, ${ }^{130}$ inhaltlich mit diesem aber ebenso verbunden ist wie die Freiheitsgewährleistungen des Binnenmarkts.

Neue Rolle des wettbewerbsverfassten Binnenmarkts? Demgegenüber erleidet die konzeptionelle Klarheit des primärrechtlichen Acquis Einbußen durch die in Abkehr vom EGV und vom VVE forcierte Vermeidung der Herausstellung der bislang elementaren Integrationsfunktion des wettbewerbsverfassten Binnenmarkts. ${ }^{131}$ Signifikant für diese Verunklarung ist bereits die textliche Abstufung der Errichtung des Binnenmarktes vom ersten operativen Zielverwirklichungsweg im EGV zu einem operativen Ziel, das nachgestellt zum Raum der Freiheit, der Sicherheit und des Rechts auftritt. ${ }^{132}$ Hingegen folgt die Reihung der internen Politiken und Maßnahmen der Union im AV der herkömmlichen Spur des EGV, indem sie vom Binnenmarkt ausgehend ${ }^{133}$ den Raum der Freiheit, der Sicherheit und des Rechts konzeptionsgenetisch und inhaltlich sinnfällig nachschaltet ${ }^{134}$ und sodann die binnenmarktflankierenden ebenso wie binnenmarktunabhängigen (aber weitgehend aus dem politischen Folgebedarf am Rande des funktionierenden Binnenmarktes erwachsenen) Politikbereiche nachführt. ${ }^{135}$ Teils verrenkt wirkende Texteingriffe leisten der konzeptionellen Verunklarung Vorschub. Neben der Nachreihung des Binnenmarkts verschwindet die Aufgabe des Systems, das den Wettbewerb innerhalb des Binnenmarkts vor Verfälschungen schützt (derzeit Art. 3 Abs.1 lit.g EGV) textlich völlig aus dem Zielbereich. Die Zielformulierung des VVE vom Binnenmarkt „mit freiem und unverfälschtem Wettbewerb“ wird nicht übernommen. Da diese Textchirurgie aber das Problem birgt, ob die zielbezogene Ab-

125 Vgl. Art. 2 EGV.

126 Zwar wird Art. 69 EGV gestrichen; andererseits wurden die einschlägigen früheren Protokolle mit Sonderbestimmungen nicht aufgehoben; dies ergibt sich aus der Liste der aufgehobenen Protokolle: vgl. Fischer: Der Vertrag von Lissabon, 2008, S. 98f.; vgl. ferner die Erklärung Irlands zu Art. 3 des Protokolls über die Position des Vereinigten Königsreichs und Irlands hinsichtlich des Raums der Freiheit, der Sicherheit und des Rechts sowie die 26. Erklärung der Konferenz zur Nichtbeteiligung eines Mitgliedstaats an einer auf den Dritten Teil Titel IV des Vertrags über die Arbeitsweise der Europäischen Union gestützten Maßnahme.

127 Vgl. Art. 14 Abs. 2 EGV; Willem Molle: The Economics of European Integration, Aldershot 1990, S. 9.

128 Vgl. Müller-Graff: Der „Raum der Freiheit, der Sicherheit und des Rechts“ im neuen Verfassungsvertrag, 2005.

129 Grundlegend EuGH Slg. 1963, 1 (van Gend \& Loos).

130 Art. 1 Ziff. 4 VvL: Art. 2 Abs. 2 EUV = projektierter neuer Art. 3 Abs. 2 EUV; s. auch Art. 2 Ziff. 34 VvL: Art. 17 Abs. 2 lit.a AV = projektierter neuer Art. 20 Abs. 2 lit.a AV. Zur unionsbürgerlichen Freizügigkeit jüngst Ferdinand Wollenschläger: Grundfreiheit ohne Markt, Tübingen 2007.

$131 \mathrm{Zu}$ dessen Integrationswirkung jüngst Hanns Jürgen Küsters: Die integrationspolitischen Wirkungen der Römischen Verträge - Eine Bilanz nach 50 Jahren, in: integration 3/2007, S. 320-332.

132 Art. 1. Ziff. 4 VvL: Art. 2 Abs. 3 EUV = projektierter neuer Art. 3 Abs. 3 EUV.

133 Vgl. Art. 2 Ziff. 41ff. VvL: Artt. 22a-59, 70-80 AV = projektierte neue Artt. 26-66, 90-100 AV.

134 Art. 2 Ziff. 64ff. VvL: Artt. 61-69h AV = projektierte neue Artt. 67-89 AV.

135 Art. 2 Ziff. 2, 77ff. VvL: Artt. 81-188r AV = projektierte neue Artt. 100-222 AV. 
rundungskompetenz des Art. $308 \mathrm{AV}$ (= projektierter neuer Art. $352 \mathrm{AV}$ ) noch zur Rechtsetzung in der Fusionskontrolle befugt, stellt erst ein Mini-Protokoll zweifelsfrei, dass der Binnenmarkt „ein System umfasst, das den Wettbewerb vor Verfälschungen schützt“ und „daß für diese Zwecke die Union erforderlichenfalls nach den Bestimmungen der Verträge, einschließlich des Artikels 308 [...] tätig wird. " Gänzlich widersinnig zu den Artt. 81-89 AV ${ }^{136}$ könnte diese Formulierung suggerieren, dass die Kommission nur gelegentlich Kartell-, Missbrauchs- und Beihilfenaufsicht betreibt. Diese Textanpassung an den Sonderwunsch eines einzigen Mitgliedstaats ist konzeptionell befremdlich. Ganz auf der Linie dieser Verunklarung liegen schließlich die Nichtübernahme der hervorgehobenen Gewährleistung der grenzüberschreitenden Marktgrundfreiheiten des Art. I-4 VVE, die Verbannung des Binnenmarktzieles aus dem Ersten Teil des AV (den Grundsätzen, ${ }^{137}$ in denen neben den Zuständigkeitsarten und -bereichen nun aber auch die Qualifikation der Tiere ,als fühlende Wesen“ verankert ist) in den Maschinenraum des Dritten Teils (Interne Politiken ${ }^{138}$ ). Dadurch wird dessen Definition sogar dem vereinfachten Änderungsverfahren des neuen Art. 48 Abs. 6 EUV zugänglich gemacht. Demgegenüber wird die Rolle der Dienste von allgemeinem wirtschaftlichen Interesse in den Grundsätzen belassen, ${ }^{139}$ obwohl deren normsystematische Rolle sich erst vor dem Hintergrund der binnenmarktrechtlichen Erfordernisse voll erschließt. ${ }^{140}$ Schließlich wird auch die Aussage gestrichen, dass die Zollunion „Grundlage der Gemeinschaft" ist. ${ }^{141}$ Konzeptionell handelt es sich bei diesen (in ihren Auswirkungen auf die Anwendung und Schaffung des Binnenmarktrechts unklaren, möglicherweise folgenlos bleibenden, möglicherweise sich folgenreich erweisenden) Text- und Lokationsänderungen des seit einem halben Jahrhundert maßgeblich bewährten Integrationsperpetuums des wettbewerblichen Binnenmarktes entweder um normative Camouflage oder epigonale Irrlichterei. Es wird rätselhaft bleiben, ob dieser Preis für den Fortgang der Reform des Primärrechts unausweichlich zu zahlen war.

Allerdings kann man gegen eine derartige Sicht einwenden, dass mit diesen Änderungen nur die Konsequenz aus einer mit der Einheitlichen Europäischen Akte begonnenen konzeptionellen Komplexierung und Transformation von einer Wirtschaftsgemeinschaft zu einem breiter angelegten Gemeinwesen gezogen wird. Seinerzeit kam es mit der Einfügung eines genuin umweltpolitischen Kapitels erstmals zu einer von marktintegrativer Begründung unabhängigen Kompetenz der EWG, ${ }^{142}$ wiewohl sie aus dem Bedürfnis nach Kompetenzabrundung am Rande der Marktintegration erwuchs. Die Verträge von Maastricht und Amsterdam vermehrten diese marktintegrativ unabhängigen Politikbereiche. Freilich steht die Fixierung auf das aufgewachsene Breitbandspektrum gemeinschaftlicher Kompetenzen in Gefahr, das gleichwohl vergleichsweise elementare Gewicht der dynamischen wirtschaftlichen Integration für die innere Haftkraft und Stabilität der Gemeinschaft zu unterschätzen. Umsomehr könnte eine solche marktabgehobene Sichtweise von den Textänderungen des Reformvertrages verstärkt werden.

136 Projektierte neue Artt. 101-109 AV; vgl. Art. 2 Ziff. 77 VvL.

137 Artt. 1a-16c AV = projektierte neue Artt. 1-17 AV; das Binnenmarktziel ist derzeit in Art. 14 Abs. 2 EGV verankert.

138 Art. 2 Ziff. 41 VvL: Art. 22a AV = projektierter neuer Art. 26 AV.

139 Art. 2 Ziff. 27 VvL: Art. $16 \mathrm{AV}=$ projektierter neuer Art. $14 \mathrm{AV}$.

140 Vgl. namentlich Art. 2 Ziff. 2 VvL: Art. 86 Abs. 2 AV = projektierter neuer Art. 106 Abs. 2 AV.

141 Art. 23 EGV.

142 Vgl. Dieter H. Scheuing: Umweltschutz auf der Grundlage der Einheitlichen Europäischen Akte, in: Europarecht (EuR) 2/1989, S. 152-192. 


\section{Der Vertrag von Lissabon im Licht der Systemkonturen des Primär- rechts}

Misst man die Neuerungen des VvL nunmehr an den zentralen Systemkonturen, die das Primärrecht unter dem Gesichtspunkt der Entwicklungsschritte der europäischen Integration in ein transnationales Gemeinwesen bislang kennzeichnen, ${ }^{143}$ wird deutlich, dass diese durch den Reformvertrag einerseits bestätigt und verstärkt, andererseits auch vermehrt werden.

\section{Bestätigung und Verstärkung primärrechtlicher Systemkonturen}

Die prägenden Systemkonturen des primärrechtlichen Bestandes erhellen sich aus den wesentlichen Charakteristika des rechtlich Neuartigen. Dieses besteht, summarisch gesprochen, in der Ablösung vom Recht klassischer völkerrechtlicher Organisationen in Richtung auf die Herausbildung eines spezifischen Rechts für ein transnationales Gemeinwesen. ${ }^{144}$ Es ist diese Transformation, die graduell konstitutionelle Bedürfnisse schuf und sich in schrittweise entstandenen, spezifischen Einzelelementen niederschlug. ${ }^{145}$ Soweit Bestimmungen des VvL diese Entwicklungsringe der Gemeinschaftsintegration betreffen, ${ }^{146}$ bauen sie auf ihnen bestätigend oder verstärkend auf.

Die Bestätigung der marktintegrativen Supranationalität: So kommt es auch mit dem VvL erneut zur Bestätigung des Ursprungsmerkmals der marktintegrativen Supranationalität. Sie wird fortgesetzt, wiewohl teils modifiziert. Es bleibt beim Grundmodell des auf völkervertraglicher Legitimationsgrundlage beruhenden außer- und nichtstaatlichen europäischen Hoheitsträgers ${ }^{147}$ mit eigener Rechtspersönlichkeit ${ }^{148}$ und substanziierter, darunter marktintegrativer Zielbindung, ${ }^{149}$ dem nach dem Prinzip der begrenzten Einzelermächtigung normbildende, administrative und judikative Befugnisse zugewiesen sind, ${ }^{150}$ dessen normbildende Kompetenzen im Rat weitgehend mit qualifizierter Mehrheitsentscheidung ausgeübt werden können, ${ }^{151}$ ohne dass der europäischen Ebene aber physische Durchsetzungsbefugnisse gegen Widerstrebende zustehen. ${ }^{152}$

Als Diskontinuität zum Acquis ist allerdings die schon vom VVE vorgesehene Streichung des Namens ,Europäische Gemeinschaft' zu registrieren. Der Gemeinschaftsbegriff, der auf eine Bezeichnungsidee von Carl Friedrich Ophüls zurückgeht, ${ }^{153}$ zählt zum semantischen Urgestein europäischer Supranationalität. Die verschmolzene Organisation erhält den Namen ,Europäische Union“. Dies ist nach dem bislang eingeschliffenen Verständnis normkategorial missverständlich, weil die umbenannte rechtspersonale europäische Organisation in ihrem Kern die Linie der supranationalen Gemeinschaft, nicht aber der inter-

143 Vgl. zu diesen signifikaten rechtlichen Merkmalen, die die EG von einer herkömmlichen internationalen Organisation abheben Müller-Graff: Primärrechtliche Entwicklungsschritte, 2007.

144 Schon EuGH Slg. 1963, 1ff. (van Gend \& Loos) nannte die Gemeinschaft eine „neue Rechtsordnung“.

145 Im Einzelnen Müller-Graff: Primärrechtliche Entwicklungsschritte, 2007.

146 So bleiben etwa die Konturelemente des Eigenmittelsystems oder der Teilhaberechte von Unionsbürgern an genuinen Hoheitsaufgaben anderer Mitgliedstaaten unberührt.

147 Vgl. im Einzelnen oben im Textabschnitt über „Konzeptionelle Kontinuität“.

148 Vgl. im Einzelnen oben im Textabschnitt über „Hauptziele des Reformvertrages“.

149 Vgl. im Einzelnen oben im Textabschnitt über „Konzeptionelle Kontinuität“.

150 Vgl. dazu unten.

151 Vgl. im Einzelnen oben im Textabschnitt über ,Steigerung des Potenzials der inneren und äußeren Handlungsfähigkeit“.

152 Vgl. dazu unten.

153 Vgl. dazu Thomas Oppermann: Juristische Fortschritte durch die europäische Integration?, in: Joachim Gernhuber (Hrsg.): Tradition und Fortschritt im Recht, Tübingen 1977, S. 415-434, hier S. 415, 419. 
gouvernementalen Unionssäulen tradiert. Andererseits ist mit der Verschmelzung jedoch nicht verbunden, dass die klassisch völkerrechtlich-intergouvernementalen Bereiche der derzeitigen zweiten Unionssäule supranationalisiert werden.

Keine konzeptionsrelevante Änderung im Merkmal der Zielbindung lässt sich daraus entnehmen, dass die Grundvorschriften über die Institutionen nunmehr im Anfangsteil des EUV massiert werden, während sie sich bislang im Wesentlichen (wiewohl nicht ausschließlich) im nachgereihten Fünften Teil des EG-Vertrages finden. ${ }^{154}$ Denn auch die reformierte Europäische Union ist nicht um der Institutionen willen geschaffen. Dies ergibt sich zum einen daraus, dass die Ziele, wie es sich für einen Gesellschaftsvertrag gehört, auch weiterhin an der Eingangspforte aufgeführt sind ${ }^{155}$ und sodann in Kompetenznormen und unmittelbar anwendbare Normen umgesetzt werden. Zum anderen findet sich die Indienststellung der Institutionen zur Verwirklichung dieser Ziele und zur Gewährleistung der Dauerhaftigkeit dieser Orientierung in dem aus dem VVE übernommenen Eingangssatz zum institutionellen Rahmen ${ }^{156}$ ausdrücklich mit den Worten hervorgehoben, dass die Union über einen institutionellen Rahmen verfügt, ,,der zum Zweck hat, ihren Werten Geltung zu verschaffen, ihre Ziele zu verfolgen, ihren Interessen, denen ihrer Bürgerinnen und Bürger und denen der Mitgliedstaaten zu dienen, die Kohärenz, Effizienz und Kontinuität ihrer Politik und ihrer Maßnahmen sicherzustellen“. Inwieweit allerdings die angesprochenen Neuerungen in der Zielformulierung ${ }^{157}$ eine Kriterienverschiebung in der Auslegung primärrechtlicher Norminhalte und im Selbstverständnis der unionalen Organe mit sich bringen, ist eine andere Frage, die noch unter dem Gesichtspunkt möglicher systemrelevanter Neuerungen anzusprechen ist. ${ }^{158}$

Eine textliche Schärfung erfährt das Prinzip der begrenzten Einzelermächtigung. Auch dieses profiliert die Union als transnationales Gemeinwesen auf völkervertraglicher Grundlage. Nicht anders als der VVE verweigert auch der VvL der Union die Kompetenzkompetenz. In textlicher Fortentwicklung des derzeitigen Primärrechts wird das Prinzip der begrenzten Einzelermächtigung im Einklang mit dem VVE nunmehr sogar ausdrücklich als solches benannt. ${ }^{159}$

Ausgebaut wird, wie gezeigt, auch das supranationale Merkmal der qualifizierten Mehrheitsentscheidung im Rat, das Entscheidungen mit Bindungswirkung in allen oder für alle Mitgliedstaaten auch dann ermöglicht, wenn nicht alle Mitgliedstaaten zustimmen. Es wird vom VvL nunmehr, unbeschadet von Ausnahmen, zum Regelprinzip erhoben, ${ }^{160}$ neudefiniert und in seiner sachgegenständlichen Anwendbarkeit teils ausgeweitet. ${ }^{161}$

Das Grundmuster des institutionellen Rahmens wird bestätigt und durch Einbeziehung der Europäischen Zentralbank auf eine nun siebenfältige Ausfächerung erweitert. ${ }^{162}$ Europäischer Rat und Kommission/Rat werden durch jeweils ein neues Amt (Präsident des Europäischen Rates; Hoher Beauftragter der Union für Außen- und Sicherheitspolitik) ${ }^{163}$ in ihrer Binnenstruktur spezifiziert. Die Verhältnisse zwischen den einzelnen Organen werden in-

154 Artt. 189ff. EGV; s. demgegenüber nur knapp Artt. 7-9 EGV und Artt. 3-5 EUV.

155 Art. 1 Ziff. 4 VvL: Art. 2 EUV = projektierter neuer Art. 3 EUV.

156 Art. 1 Ziff. 14 VvL: Art. 9 EUV = projektierter neuer Art. 13 EUV.

157 Vgl. im Einzelnen oben im Textabschnitt über „Konzeptionelle Komplexierung“.

158 S. unten.

159 Art. 1 Ziff. 6 VvL: Art. 3b Abs. 1 S. 1 und Abs. 2 EUV = projektierter neuer Art. 5 Abs. 1 S. 1 und Abs. 2 EUV.

160 Vgl. Art. 1 Ziff. 17 VvL: Art. 9c Abs. 3 EUV = projektierter neuer Art. 16 Abs. 3 EUV; vgl. dazu Art. III-23 Abs. 3 VVE.

161 Vgl. im Einzelnen oben im Textabschnitt über, „Steigerung des Potenzials der inneren und äußeren Handlungsfähigkeit“.

162 Vgl. Art. 1 Ziff. 14 VvL: Art. 9 Abs. 1 EUV = projektierter neuer Art. 13 Abs. 1 EUV.

163 Vgl. oben im Textabschnitt über die „Steigerung des Potenzials der inneren und äußeren Handlungsfähigkeit“. 
nerhalb des aufgewachsenen Systemrahmens detailliert fortentwickelt, ${ }^{164}$ ohne dies hier im Einzelnen aufgreifen zu können. Eine physische Durchsetzungsbefugnis gegen Widerstrebende bleibt, wie schon angesprochen, dem europäischen Hoheitsträger versagt und residiert damit in der alleinigen Hoheit der Mitgliedstaaten. Diese Grenze wird auch nicht überschritten durch die aufgezeigten Neuerungen betreffend Europol, Eurojust, Schaffung einer Europäischen Staatsanwaltschaft, Mindestvorschriften im Strafbereich oder zur gegenseitigen Anerkennung gerichtlicher Urteile und Entscheidungen in Strafsachen. ${ }^{165}$ Unionale Normsetzung, Normadministration und Normauslegung kann mithin auch weiterhin nur mittels mitgliedstaatlicher Hoheitsgewalt gegen Widerstrebende durchgesetzt werden. Auch die reformierte Union ist daher wie derzeit auf die pflichtgemäße Loyalität der Mitgliedstaaten ${ }^{166}$ angewiesen.

Ausbau der subjektiven Rechte: Es zählt zu den markanten Neuerungen des VvL, dass er das im Gang der Gemeinschaftsintegration geschaffene zweite herausragende Strukturelement, nämlich die Anerkennung subjektiver Rechte auf völkervertraglicher Grundlage (Marktgrundfreiheiten ${ }^{167}$ mit Entstehung einer transnationalen Privatrechtsgesellschaft, ${ }^{168}$ Unionsbürgerrechte, Gemeinschaftsgrundrechte) im Grundrechtsbereich textlich mittels der Erhebung der Grundrechte-Charta zu einem vertragsgleichen Text ${ }^{169}$ verstärkt.

Britannien und Polen begrenzen jedoch die Rechtswirkung der Charta, soweit es um nationales Rechts geht, das nach der Charta (begrenzt) überprüft werden kann. Ob durch deren Formulierung die sogenannte Erstreckungsrechtsprechung des EuGH zu den judikativen Gemeinschaftsgrundrechten schon allgemein zurückgeschnitten wird, ist umstritten und hier nicht zu vertiefen. ${ }^{170}$ Art. 1 des Protokolls über die Anwendung der Charta der Grundrechte der Europäischen Union auf Polen und das Vereinigte Königreich bestimmt, dass die Charta keine Ausweitung der Befugnis des EuGH oder eines polnischen oder britischen Gerichts bewirkt, um festzustellen, dass die Rechts- und Verwaltungsvorschriften, die Gepflogenheiten oder das Handeln Polens oder Britanniens nicht mit den durch die Charta bekräftigten Grundrechten, Freiheiten und Grundsätzen in Einklang stehen. Ob speziell von Art. 1 auch die bisherige Erstreckungsrechtsprechung des EuGH zu den judikativen Gemeinschaftsgrundrechten betroffen ist, ist unklar. Dagegen spricht, dass lediglich eine Ausweitung der Befugnisse des EuGH ausgeschlossen wird, um die es aber bei der fortsetzenden Ausübung vorhandener Befugnisse und der daraus erwachsenen Rechtsprechung nicht geht. Diese Sonderheit der beiden Staaten im Vergleich zu den 25 anderen Mitgliedstaaten erstaunt nicht für Britannien, das sich seit jeher mit dem Gedanken der Überprüfung nationaler Maßnahmen anhand europäischer Grundrechtestandards (und im Blick auf die Charta vielleicht nicht ohne Grund) ${ }^{171}$ schwertat.

164 Vgl. z.B. der Übergang vom Erfordernis der Anhörung des Europäischen Parlaments in Art. 308 EGV zum Zustimmungserfordernis in Art. 2 Ziff. 289 VvL: Art. 308 AV = projektierter neuer Art. 352 AV.

165 Art. 2 Ziff. 67 VvL: Art. 69a AV = projektierter neuer Art. 82 AV; vgl. dazu Art. III-270 VVE.

166 Art. 1 Ziff. 5 VvL: Art. 3a Abs. 3 EUV = projektierter neuer Art. 4 Abs. 3 EUV; vgl. dazu Art. I-5 Abs. 2 VVE und derzeit Art. 10 EGV.

167 Grundlegend EuGH Slg. 1963, 1ff. (van Gend \& Loos).

168 Vgl. dazu Peter-Christian Müller-Graff: Die Europäische Privatrechtsgesellschaft in der Verfassung der Europäischen Union, in: Peter-Christian Müller-Graff/Herbert Roth (Hrsg.): Recht und Rechtswissenschaft, Heidelberg 2000, S. 271-306 mit weiteren Nachweisen.

169 Vgl. oben im Textabschnitt „Erhöhung der Legitimation unionaler Maßnahmen“.

170 Vgl. als Hinweise zur Diskussion Fischer: Der Vertrag von Lissabon, 2008, S. 116; s. auch Peter-Christian Müller-Graff: Das Verhältnis von Grundrechten und Grundfreiheiten im Lichte des Europäischen Verfassungsvertrags, in: Jürgen Schwarze (Hrsg.): Europäische Verfassung und Grundrechtecharta, Europarecht (EuR) Beiheft 1/2006, S. 19-42, hier S. 19, 31f.

171 Zu den Problemen der Charta vgl. jüngst Stein: Haben die Engländer nicht eigentlich recht?, 2008, S. 37-43. 
Sachgegenständliche Ausweitung der unmittelbaren Anwendbarkeit und des Vorrangprinzips: Im Hinblick auf die beiden Systemkonturen der unmittelbaren Anwendbarkeit von Gemeinschaftsrecht ${ }^{172}$ und des Vorrangprinzips ${ }^{173}$ enthält der VvL rechtsprinzipiell weder Abstriche noch Neuerungen. ${ }^{174}$ Insbesondere enthalten die in der derzeitigen zweiten und dritten Säule lozierten primärrechtlichen Bestimmungen durch den Reformvertrag keine zur unmittelbaren Anwendbarkeit geeigneten Normen, sondern durchweg Ermächtigungsnormen.

Allerdings kann es in dem Bereich, der derzeit in der dritten Unionssäule geregelt ist, nunmehr, soweit eine entsprechende Ermächtigung besteht, zu einer unmittelbaren Anwendbarkeit von Sekundärrechtsakten in Gestalt von Verordnungen und (ausnahmsweise) Richtlinien, aber auch Beschlüssen im Rahmen der einschlägigen Rechtsprechung des EuGH kommen. Der derzeitige ausdrückliche Ausschluss der unmittelbaren Anwendbarkeit von Rahmenbeschluss und Beschluss durch Art. 34 Abs. 2 lit.b und c EUV fällt zusammen mit den Sonderrechtsakttypen der dritten Säule weg. Sachgegenständlich erfährt damit auch das Vorrangprinzip eine Ausdehnung auf Rechtstexte in den Bereichen, die vom VvL aus der Intergouvernementalität in die Supranationalität überführt werden. Dies betrifft namentlich die polizeiliche und justitielle Zusammenarbeit in Strafsachen.

Beibeihaltung der Legitimationsquelle der Mitglieder des Europäischen Parlaments: In der Frage der Legitimationsgrundlage der Mitglieder des Europäischen Parlaments wechselt der VvL die derzeitige Formulierung ,Vertreter der Völker der in den Mitgliedstaaten zusammengeschlossenen Staaten“ in die Benennung als „Vertreter der Unionsbürgerinnen und Unionsbürger“. Jedoch beinhaltet das textliche Verschwinden der Völker nicht auch die Beseitigung des Legitimationsrückgriffs auch auf diese. Denn die Direktwahl erfolgt weiterhin erstens nach den jeweiligen mitgliedstaatlichen Regeln und zweitens vor allem im Rahmen von Länderkontingenten, die nicht nach dem demokratischen Prinzip der repräsentativen Gleichwertigkeit jedes Unionsbürgers fixiert sind. Das derzeitige Prinzip der degressiven Proportionalität ${ }^{175}$ wird fortgeschrieben und nunmehr im Einklang mit dem VVE ausdrücklich als solches benannt. ${ }^{176}$ Es ist zugleich ein demokratietheoretischer Grundmakel.

Ausweitung der marktintegrationsunabhängigen Supranationalitätsgegenstände: Die Systemkontur der marktintegrationsunabhängigen Supranationalitätsgegenstände erfährt durch den VvL eine bemerkenswerte Stärkung. Dies verdankt sich zum einen der weitgehenden Übernahme der Gegenstände der derzeitigen dritten Säule in die Supranationalität. Zugleich wird nunmehr der gesamte Raum der Freiheit, der Sicherheit und des Rechts von dem Erfordernis gelöst, die Binnenmarktintegration zu fördern. ${ }^{177}$ Des Weiteren werden weitere, nicht notwendig marktintegrativ erforderliche Sachaufgaben ausdrücklich in den supranationalen Aktionsradius eingestellt. ${ }^{178}$

Stärkung des übergreifenden Rahmens für gemeinschaftliche und außergemeinschaftliche Aufgaben: Auch das Konturelement des vom Vertrag von Maastricht kreierten übergreifenden Rahmens von Zielen, Werten und Institutionen für gemeinschaftlich-supranationale und außergemeinschaftlich-intergouvernementale Aufgaben und Politiken erfährt eine bemerkenswerte Fortentwicklung. Mit der Überwindung der Zweiteilung in Europäische

172 Grundlegend EuGH Slg. 1963, 1 (van Gend \& Loos).

173 Grundlegend EuGH Slg. 1964, 1251 (Costa/ENEL).

174 Vgl. zum Vorrangprinzip schon oben im Textabschnitt über „Relativer Rückschnitt von Texttransparenz“.

175 Vgl. in der Sache Art. 190 Abs. 2 EGV.

176 Vgl. oben im Textabschnitt „Erhöhung der Legitimation unionaler Maßnahmen“.

177 Vgl. derzeit beispielsweise namentlich Artt. 61 lit.a, 65 EGV.

178 Vgl. oben im Textabschnitt „Steigerung des Potenzials innerer und äußerer Handlungsfähigkeit“. 
Gemeinschaft und Europäische Union erledigt sich die Aufteilung oder Verdoppelung dieser Vorschriften. Folgerichtig ersetzt ein einziger Zielartikel die bisherigen Zielartikel von EGV und EUV, ohne allerdings die konkretisierende Nachführung der Ziele in den einzelnen Sachkapiteln von EUV und AV aufzuheben. Im Gegenteil kommt es in der Tiefe des Textes zu einem Aufwuchs von Einzelzielen. ${ }^{179}$ Konsequent umfasst der neue Werteartikel die gesamte Spannbreite der neu konfigurierten Unionsgemeinschaft. Schlüssig werden im Anschluss an den VVE die derzeit auf EUV und EGV verteilten Funktions- und Institutionsbestimmungen des sogenannten einheitlichen institutionellen Rahmens ${ }^{180}$ in einen profilbildenden Institutionenabschnitt im EUV zusammengeführt. ${ }^{181}$ Zwar werden alle Organe schlüssig in den Dienst der Ziele der Union gestellt, ${ }^{182}$ doch wird jedem Organ, insbesondere auch der Europäischen Zentralbank, konkretisierend ihre jeweils spezifische Funktionsaufgabe zugewiesen. So kann insbesondere das vorrangige Ziel des Europäischen Systems der Zentralbanken, die Preisstabilität zu gewährleisten, ${ }^{183}$ nicht unabhängig von den Voraussetzungen zur Unterstützung der allgemeinen Wirtschaftspolitik über die allgemeine Zielbindung ausgehebelt werden. Folgerichtig zum Aufbau der Union von ihren Sachaufgaben her erfolgt die Feinjustierung der Aufgaben jedes Organs schließlich im jeweiligen sachpolitischen Kapitel. Insgesamt ist aber die Methode des VvL geeignet, durch die Bündelung der profilbildenden Ziel-, Wert- und Institutionen-Bestimmungen die Perzeption der Union als Gemeinwesen mit Gemeinwohlausrichtung, konkretisierenden Zielen, Kompetenzen und Institutionen zu stärken.

Stärkung der Repräsentanzgleichwertigkeit der Unionsbürger: Schließlich kommt es zu einem großen Entwicklungssprung beim primärrechtlich in Art. 205 Abs. 4 EGV angelegten Systemelement der demokratietheoretisch stimmigen Gleichwertigkeit der Repräsentanz der Unionsbürger im europäischen politischen Entscheidungsverfahren. Sie erfolgt zwar, wie gesehen, nicht über die Zusammensetzung des Europäischen Parlaments. Wohl aber spiegelt sich die politische unionsbürgerliche Gleichwertigkeit in der Neudefinition der qualifizierten Mehrheit als doppelte Mehrheit im Rat. ${ }^{184}$

\section{Neue rechtliche Systemkonturen des transnationalen Gemeinwesens?}

Fragt man abschließend, ob der VvL seinerseits markante innovative primärrechtliche Systemkonturen des transnationalen Gemeinwesens schafft, so fallen bei einer vorläufigen ersten Betrachtung mehrere Elemente auf, ohne mit diesen aber die Tiefe des Textraums voll auszuschöpfen. Immerhin lässt sich aber erkennen, dass an beiden Polen des europäischen Zusammenwirkens Neuerungen sichtbar werden: in der Transnationalisierung und in der unionalen Zusammenführung.

Neue Systemkonturen der Transnationalisierung: In dem spezifisch transnationalen Aspekt der Gemeinschaftsbildung markieren weder die Einführung der Kompetenzsystematik noch die Erleichterung der Verstärkten Zusammenarbeit zwischen einer Teilmenge von Mitgliedstaaten ${ }^{185}$ kategorial neue Systemelemente. Sie sind lediglich Ausdruck einer zusätz-

179 So namentlich für das auswärtige Handeln der Union.

180 Derzeit Artt. 3-5 EUV i.V.m. Artt. 189ff. EGV.

$181 \mathrm{Vgl}$. oben.

182 Art. 1 Ziff. 14 VvL: Art. 9 EUV = projektierter neuer Art. 13 EUV.

183 Art. 2 Ziff. 91 VvL: Art. 105 Abs. 1 S. 1 AV = projektierter neuer Art. 127 Abs. 1 S. 1 AV; vgl. dazu Art. 105 Abs. 1 S. 1 EGV.

184 Vgl. oben im Textabschnitt über „Erhöhung der Legitimation unionaler Maßnahmen“.

185 Art. 1 Ziff. 22 VvL: Art. 10 EUV = projektierter neuer Art. 20 EUV; Art. 2 Ziff. 278 VvL: Artt. 280a-280i AV = projektierte neue Artt. 326-334 AV; vgl. dazu Art. I-44 VVE; Artt. III-416-III-420 VVE. 
lichen Reifung bereits früher eingeführter Konturen der föderationsähnlichen Kompetenzordnung und der vertragsrechtlichen Fundierung. Auch die Nichtteilnahme Britanniens und Polens an der (begrenzten) Überprüfung inländischer Maßnahmen mittels der GrundrechteCharta ist nur ein weiterer Fall der Nichtteilnahme einzelner Mitgliedstaaten an einem primärrechtsverbindlichen Integrationsschritt.

Hingegen bildet vor allem die neuartige primärrechtliche Einbeziehung der nationalen Parlamente oder deren Kammern in die politische und judikative Architektur der Union ${ }^{186}$ ein innovatives Systemelement im transnationalen Charakter der Gemeinschaftsintegration. Denn mit diesen zarten Ansätzen der Einbindung nationaler Parlamente kann sich im unionalen Entscheidungsverfahren verfahrensförmlich auch der mitgliedstaatliche Parlamentarismus artikulieren. Die Letztverantwortlichkeit der unionalen Organe einschließlich des Europäischen Parlaments für die unionale Rechtsetzung wird dadurch nicht aufgehoben, aber doch die Aufmerksamkeit des lebendigen, im jeweiligen Mitgliedstaat wirkenden Parlamentarismus auf die unionale Rechtsetzung gelenkt. Insbesondere im Frühwarnsystem liegen Chancen für die innere Kohäsion und Akzeptanz der Union und ihrer Maßnahmen: so insbesondere darin, dass unionale Maßnahmen von den zuständigen Organen mit angereichertem Sinn für die mitgliedstaatliche Vielfalt gestaltet werden oder unterbleiben. Aber auch die fehlende Repräsentanz aller mitgliedstaatlicher Staatsangehörigkeiten in der verkleinerten Kommission kann durch die vorgesehene frühzeitige Einbeziehung der nationalen Parlamente ausgeglichen werden, wenn sich die Kommission hinreichend reaktionsfähig und dialogbereit zeigt. Sie mag in den mit öffentlicher Legitimation ausgestatteten Stellungnahmen nationaler Parlamente zu unionalen Rechtsetzungsvorhaben sogar eine Hilfe in ihrer Diskussion mit Vertretern einzelner Interessenverbände finden. Man mag sich hierbei erinnern, dass die Einbeziehung der Mitglieder nationaler Parlamentarier im Konvent ${ }^{187}$ sich in der Gesamtbetrachtung als kohäsionsförderlich bei der Diskussion, Lösungsfindung, Textausarbeitung und innenpolitischen Vertretung des Verfassungsvertrages erwiesen hat.

Als ein zweites, die Transnationalität betonendes neues Strukturelement der Gemeinschaftsbildung lässt sich die ausdrückliche Zuerkennung eines voraussetzungslosen Austrittsrechts jedes Mitgliedstaats erkennen. Es ist nicht einmal von der Erfüllung der Voraussetzungen der „clausula rebus sic stantibus“ abhängig. Dass sich aus dessen Existenz indes uniformierungsdämpfende Auswirkungen auf die unionale Politik ergeben, ist freilich eher zweifelhaft.

Neue Systemkonturen unionaler Zusammenführung: In dem spezifisch gemeinwesenbildenden Aspekt der Integration mag man manchen Neuerungen vielleicht nur eine graduelle Ausreifung bereits vorhandener Systemkonturen zuerkennen: so gewiss der Aufwertung der Grundrechte-Charta, aber wohl auch der legitimationssteigernden Neudefinition der qualifizierten Mehrheit als doppelte Mehrheit ${ }^{188}$ mit ihrer Überwindung des legitimationstheoretisch nicht sinnfällig begründbaren Art. 205 Abs. 2 EGV; denn das dadurch anerkannte demografieabhängige Einflussgewicht eines Mitgliedstaats findet sich ansatzweise schon im geltenden Recht als Verhinderungshandhabe, ${ }^{189}$ das nun aber auch bei der Gestaltung zum Tragen kommt.

Als innovative gemeinwesenbildende Neuerungen dürfen indes mehrere institutionelle Neuerungen zur Stärkung der unionalen Gemeinwohlorientierung ${ }^{190}$ gelten: die Einführung

186 Vgl. dazu im Einzelnen oben Textabschnitt zu „Erhöhung der Legitimation unionaler Maßnahmen“.

187 Vgl. Peter-Christian Müller-Graff: Der Europäische Verfassungskonvent, Wien 2004, S. 16f.

188 Vgl. oben im Textabschnitt über „Erhöhung der Legitimation unionaler Maßnahmen“.

189 Vgl. Art. 205 Abs. 4 EGV.

190 Vgl. zur Frage des europäischen Gemeinwohls Gerold Ambrosius/Peter Schmitt-Egner (Hrsg.): Europäisches Gemeinwohl - Historische Dimension und aktuelle Bedeutung, Baden-Baden 2006. 
des Amts des Präsidenten des Europäischen Rats, das nicht mit der Ausübung eines einzelstaatlichen Amtes verbunden sein darf; ${ }^{191}$ die Aufhebung des Prinzips der Repräsentanz aller mitgliedstaatlichen Staatsangehörigkeiten in der Kommission; ${ }^{192}$ die (vorsichtig auf parteipolitische Stärkeverhältnisse abstellende) Berücksichtigungspflicht des Ergebnisses der Wahlen zum Europäischen Parlament beim Vorschlag des Kandidaten für das Amt des Kommissionspräsidenten durch den Europäischen Rat; ${ }^{193}$ die organüberbrückende, aber möglicherweise nicht konstruktiv ausfüllbare Doppelbindung des Amtes des Hohen Vertreters der Union für die Außen- und Sicherheitspolitik an Rat (Vorsitz ,Auswärtige Angelegenheiten') und Kommission (einer der Vizepräsidenten). ${ }^{194}$ Auch die Regularisierung der Konventsmethode für Vertragsänderungen im ordentlichen Verfahren ${ }^{195}$ lässt sich hier einreihen.

In ihrem wirkungsrelevanten Innovationsgrad für die Gemeinschaftsbildung unklar ist demgegenüber die Neuformulierung der unionalen Zielbindung im Zielartikel. ${ }^{196}$ Zwar gibt die Ausrichtung auf Frieden, Werte und Wohlergehen im Wesentlichen nur den Acquis ${ }^{197}$ wieder. Indes könnte die textliche Voranstellung des Raums der Freiheit, der Sicherheit und des Rechts unter Nachreihung des Binnenmarktziels und der Wegfall des seit Beginn bestehenden Introitus des EG-Vertrages, dass es „Aufgabe der Gemeinschaft ist [...], durch die Errichtung des Gemeinsamen Marktes“ und zwei weitere Wege die nachfolgenden Ziele zu fördern, ${ }^{198}$ eine Umorientierung des primären Verflechtungswegs über die Wirtschaft auf ein Zusammenwachsen über einen staatsähnlich befriedeten, rechtlich gesicherten Raum andeuten. Dies bleibt aber fraglich, da letzterer ohne den Sockel des binnenmarktlichen Freiheitsraums kaum einen hinreichenden Selbststand und eine entsprechende transnationale Haftkraft aufweisen dürfte, mithin einer Rekonstruktion unter Einschluss des Binnenmarktes bedarf. ${ }^{199}$ Spekulativ bleibt, ob das herausgehobene Ziel der Werteförderung ${ }^{200}$ in derselben Weise wie das Friedens- und Prosperitätsziel den Zusammenhalt antreibt. Dies gilt ähnlich für die aus dem Verfassungsvertrag verbliebenen Restbestände staatsanalogisierender Semantik („Gesetzgebungsverfahren“).

\section{Zusammenfassung}

Zusammenfassend ist festzuhalten, dass der Lissabonner Vertrag in seiner primärrechtlichen Substanz nicht hinter dem Verfassungsvertrag zurückbleibt. Zwar leistet er nicht dessen kohärente Kodifikation des Primärrechts. Auch birgt er konzeptionelle Unklarheiten. Aber insgesamt verstärkt er die Normstruktur und Normwirkung des transnationalen Gemeinwesens sowohl in deren transnationalen wie in deren zusammenführenden Zügen. Dies geschieht überwiegend durch ein systemstimmiges Ansetzen an den bereits vorhandenen normativen Spezifika des transnationalen europäischen Gemeinwesens, aber auch durch deren zeitgerechte Erweiterungen. Die Reform bietet eine gute Chance, die mehr als 50-jährige Erfolgsgeschichte der europäischen Integration in die Zukunft fortzusetzen.

191 Art. 1 Ziff. 16 VvL: Art. 9b Abs. 5 a.E. EUV = projektierter neuer Art. 15 Abs. 5 a.E. EUV; vgl. dazu Art. I-22 Abs. 3 VVE.

192 Vgl. dazu oben im Textabschnitt „Steigerung des Potenzials der inneren und äußeren Handlungsfähigkeit“.

193 Ebenda.

194 Art. 1 Ziff. 19 VvL: Art. 9e Abs. 3 und 4 EUV = projektierter neuer Art. 18 EUV; vgl. dazu Art. I-28 VVE.

195 Art. 1 Ziff. 56 VvL: Art. 48 Abs. 3 EUV = projektierter neuer Art. 48 Abs. 3 EUV.

196 Vgl. dazu oben im Textabschnitt „Konzeptionelle Komplexierung“.

197 Vgl. dazu Peter-Christian Müller-Graff: Die Einheit des Primärrechts nach Amsterdam: Einheit und Kohärenz der Vertragsziele von EG und EU, in: Armin von Bogdandy/Claus-Dieter Ehlermann (Hrsg.): Konsolidierung und Kohärenz des Primärrechts nach Amsterdam, Europarecht (EuR) Beiheft 2/1998, S. 6780.

198 Art. 2 EGV.

199 Vgl. oben.

200 Art. 1 Ziff. 3 und 4 VvL: Artt. 1a, 2 EUV = projektierte neue Artt. 2, 3 EUV. 